\title{
The Virtual Fields Method for Extracting Constitutive Parameters From Full-Field Measurements: a Review
}

\author{
M. Grédiac*, F. Pierron ${ }^{\dagger}$, S. Avril ${ }^{\dagger}$ and E. Toussaint* \\ * Laboratoire de Mécanique et Ingénieries, Université Blaise Pascal Clermont II-IFMA, Aubière, France \\ † Laboratoire de Mécanique et Procédés de Fabrication, Ecole Nationale Supérieure d'Arts et Métiers, Châlons-en-Champagne, France
}

\begin{abstract}
The virtual fields method has been developed for extracting constitutive parameters from full-field measurements provided by optical non-contact techniques for instance. It is based on the principle of virtual work written with some particular virtual fields. This paper can be regarded as a general review summarising some 15 years of developments of this method. The main aspects of the method are first recalled in the case of both linear and non-linear constitutive equations. They are then illustrated by some recent relevant examples. Some studies underway as well as relevant issues to be addressed in the near future are eventually discussed.
\end{abstract}

KEY WORDS: heterogeneous tests, identification, inverse problem, virtual fields method

\section{Introduction}

The practical identification of the parameters governing the constitutive equations of structural materials is a key issue in experimental solid mechanics. It usually relies on performing some simple mechanical tests such as tensile or bending tests. In these cases, a closed form solution for the corresponding mechanical problem provides a direct link between unknown parameters and measurements which are usually local strain components and applied loads. However, such a classical approach suffers from two main drawbacks which can be summarised as follows:

- The assumptions needed to link directly the unknown parameters to the load and strains are usually rather stringent (uniform pressure distribution at the specimens' ends for the uniaxial tensile test, for instance). These requirements are particularly difficult to meet for anisotropic materials [1, 2], for instance. When heterogeneous materials are tested (weld, functionally graded material, etc.), it is even more critical;

- Only a small number of parameters can be determined with such mechanical tests, hence the need to perform several tests when the constitutive equations depend on more parameters than isotropy (anisotropic elasticity, plasticity for instance).
This statement has led several authors to consider alternative approaches based on the processing of heterogeneous strain fields. In this case, both limitations can be overcome. Indeed, if the test is well designed, the applied loading gives rise to a heterogeneous strain field which involves the whole set of constitutive parameters. Their simultaneous identification is therefore possible provided that the heterogeneous field is measured with some suitable non-contact whole-field measurement technique and that a robust identification strategy extracting the parameters from this type of measured data is available. Full-field measurement techniques such as digital image correlation, speckle and moiré interferometry or grid method have recently spread in the experimental mechanics community, thus leading their users to wonder about the use of such measurements for identification purpose. The availability of identification strategies enabling the extraction of the constitutive parameters is in fact a key issue in such approaches as there is generally no closed-form solution allowing a direct link between measurements and unknown parameters. Different approaches have been proposed in the recent past among which the so-called virtual fields method (VFM). This paper summarises the 15-year developments of the method. It will give the main features of this approach and illustrate its relevancy through a review of some recent examples of application. One 
of its objectives is also to raise interest from the mechanics of materials community that is gradually becoming more and more aware of the potential of such approach to address difficult mechanical characterisation issues.

An overview of the problem is proposed in the first section. The general principles of the VFM are then given and developed. Two cases are clearly distinguished: constitutive equations depending linearly on the constitutive parameters and non-linear constitutive equations. In the first case, some suitable strategies have been proposed by the co-authors in the recent past to determine the virtual fields (this determination is in fact a key point in the method). These strategies are recalled and explained in the following sections. A first attempt at a non-linear behaviour (elasto-plasticity) is also presented together with various experimental implementations of the method.

\section{Overview of the Problem}

\section{I. Statement of the problem}

Let us consider the problem of identification of parameters governing constituting equations. In such a problem, the geometry of the specimen is known. 3D volume displacement fields inside the solid are measured with a suitable technique such as tomography or the displacement field is provided by a suitable optical non-contact technique on the external surface only. Note that the strain field can be deduced from the displacement field by numerical differentiation. The applied loading is measured with a load cell. The type of constitutive equations is chosen a priori for its relevancy and the objective here is to determine the parameters which govern the constitutive equations. The main difficulty comes from the fact that the measured displacement or strain components are generally not directly related to the unknown parameters. In other words, no closed-form solution for the displacement, strain and stress fields is available. The stress, strain and displacement components are linked through the wellknown equations of continuum mechanics, namely the equations of equilibrium, the strain/displacement relations and the constitutive equations. These equations are verified at any point of the specimen, but they do not provide any local link between measurements and unknown parameters. Such a problem is often referred to as inverse in the literature while the direct problem is the usual determination of the strain/displacement/stress fields assuming that the constitutive equations, the constitutive parameters, the loading and the geometry are known.

\subsection{Different tools to solve the problem}

Various methods have been recently proposed in the literature to solve this inverse problem, namely the finite element model updating technique [3], the constitutive equation gap method [4], the equilibrium gap method [5], the reciprocity gap method [6] and the VFM [7]. The most popular one is certainly the finite element model updating technique which has been used for identifying parameters driving linear [8] and mainly non-linear constitutive equations [9-16]. It consists in constructing a finite element model of the mechanical test, collecting displacement or strain components at some nodes and building up a cost function with the difference between numerical and experimental displacements at these nodes. Minimising this cost function with respect to the unknown constitutive parameters provides the solution of the problem. It must be noted at this stage that this method is very general and flexible. In particular, it does not specifically require full-field measurements.

This approach exhibits however a number of shortcomings. First, it is iterative and requires the solution of a direct calculation for each evaluation of the cost function. Also, the loading distribution must be known to feed the finite element model whereas only the resulting force is generally measured, thus leading to some assumptions regarding this distribution. If these assumptions are incorrect, a bias on the identified parameter will exist. An alternative is to use the measured displacements obtained by fullfield measurements at the boundary of the active area as input boundary conditions in the finite element model. However, some equation involving the forces applied to the specimen is required in addition to be able to identify the full set of stiffness components, for instance. And also, the noise on the displacement measurements will affect the updating routine and may cause some additional bias.

The development of the VFM has been targeted at avoiding these drawbacks by taking maximum advantage of the availability of full-field measurements.

\section{The VFM: General Principle}

The VFM is based on the principle of virtual work [17]. This principle can be written as follows for a given solid of volume $V$

$$
-\int_{V} \sigma: \varepsilon^{\star} \mathrm{d} V+\int_{S_{f}} \overline{\mathbf{T}} \cdot \mathbf{u}^{\star} \mathrm{d} S+\int_{V} \mathbf{f} \cdot \mathbf{u}^{\star} \mathrm{d} V=\int_{V} \rho \gamma \cdot \mathbf{u}^{\star} \mathrm{d} V
$$




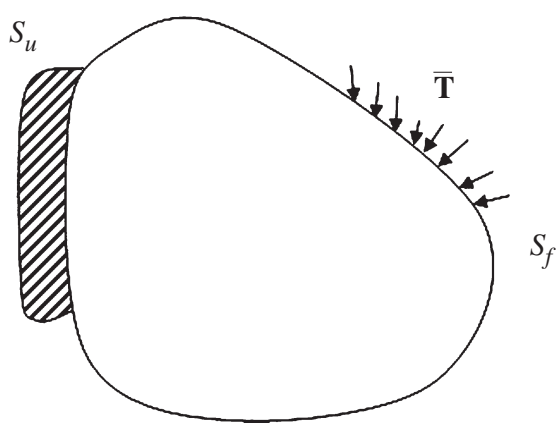

Figure I: Solid of any shape loaded on its boundary

where $\sigma$ is the actual stress tensor, $\varepsilon^{\star}$ is the virtual strain tensor, $\overline{\mathbf{T}}$ is the distribution vector of loading tractions acting on the boundary, $S_{f}$ is the part of the solid boundary where the tractions are applied (Figure 1), $\mathbf{u}^{\star}$ is the virtual displacement vector, $\mathbf{f}$ is the distribution of volume forces acting on $V, \rho$ is the mass per unit volume and $\gamma$ the acceleration.

A virtual displacement field is actually a test function for which the previous equation is verified, and a virtual strain tensor is a strain tensor derived from a given virtual displacement. An important feature is the fact that the above equation is verified for any kinematically admissible virtual field $\left(\mathbf{u}^{\star}, \varepsilon^{*}\right)$. Kinematically admissible means that $\mathbf{u}^{\star}$ must be continuous across the whole volume and it must be equal to the prescribed displacement on the boundary $S_{u}$ where displacements are prescribed (Figure 1). But this condition is the only one required for fulfilling Equation (3.1). At this stage, the constitutive equations are introduced. One can write in the general case

$\sigma=g(\varepsilon)$

where $g$ is a given function of the actual strain components. The constitutive parameters are also involved in $g$. Equation (3.1) can be written therefore in this case:

$-\int_{V} g(\varepsilon): \varepsilon^{\star} \mathrm{d} V+\int_{S_{f}} \overline{\mathbf{T}} \cdot \mathbf{u}^{\star} \mathrm{d} S=\int_{V} \rho \gamma \cdot \mathbf{u}^{\star} \mathrm{d} V$.

It is a trivial matter to see that any new virtual field in Equation (3.3) leads to a new equation involving the constitutive parameters provided that the actual strain field is heterogeneous. The VFM relies on this important property. It is based on Equation (3.3) written with a given set of virtual fields [7]. This set of equations is used to extract the unknown constitutive parameters.

The choice of the virtual fields is a key issue in the method. Their number and their type depend in fact on the nature of $g$ in Equation (3.2). Two cases must be distinguished:

- in the first case, such as linear elasticity, some cases of damage or non-linear elasticity, the constitutive equations depend linearly on the constitutive parameters. It will be shown below that in this case, writing Equation (3.3) with as many virtual fields as unknowns leads to a linear system which directly provides the parameters after inversion, provided that the actual strain field is heterogeneous and the virtual fields are independent. Note that linear viscoelasticity is included in this case but the virtual fields must be complex, as shown in Section 8. The choice of the virtual fields follows in this first case a precise strategy discussed in Section 4.

- in the second case, the constitutive equations are not linear functions of the constitutive parameters. This case occurs in elasto-plasticity for instance. The identification strategy relies in this case on the minimisation of a residual constructed with Equation (3.3), as illustrated in Section 9.

\section{Case of Constitutive Equations Depending Linearly on the Constitutive Parameters: Choice of the Virtual Fields}

\section{I. Introduction}

The case of constitutive equations linearly depending on the constitutive parameters is important as the latter may be directly retrieved in this case. The following subsections describe some possible choices for the virtual fields in this case: virtual fields chosen by hand, so-called special virtual fields and eventually special virtual fields which minimise noise effects. Let us choose the example of linear elasticity for this purpose. In this case and using the usual contracted rule for the indices, the constitutive equations can be written

$\sigma_{i}=Q_{i j} \varepsilon_{j}, \quad(i, j) \in[1,2, \ldots, 6]$.

Introducing the above constitutive equations in Equation (3.1) where the case of statics is considered for the sake of simplicity ( $\gamma=0$ in Equation 3.3), the principle of virtual work may be written as:

$\int_{V} Q_{i j} \varepsilon_{j} \varepsilon_{i}^{*} \mathrm{~d} V=\int_{S_{f}} \overline{\mathbf{T}} \cdot \mathbf{u}^{*} \mathrm{~d} S$.

If the material is heterogeneous, it is possible to parameterise the spatial dependance of the stiffness components with respect to the space variables, 
either in a discrete or continuous way (see for instance Grédiac et al. [18], and Section 10). Here, for the sake of simplicity, let us assume that all the $Q_{i j}$ values are constant across $V$. Therefore,

$Q_{i j} \int_{V} \varepsilon_{j} \varepsilon_{i}^{*} \mathrm{~d} V=\int_{S_{f}} \overline{\mathbf{T}} \cdot \mathbf{u}^{*} \mathrm{~d} S$.

Any new virtual field provides a new linear equation of the type of Equation (4.3), then writing this equation with as many virtual fields as unknowns eventually provides the following linear system where the constitutive parameters are unknown

$\mathbf{P Q}=\mathbf{R}$,

where $\mathbf{P}$ is a square matrix, $\mathbf{Q}$ a vector whose components are the $Q_{i j} s$ and $\mathbf{R}$ is a vector whose components are the virtual works of the applied forces computed for the whole set of virtual fields. It can be checked that the equations in system 4.4 are independent provided that the actual strain field is heterogeneous and the virtual strain fields independent [18]. This approach was the only one available until the special virtual fields presented below. With such an approach, the question of the optimal choice of the virtual fields remains open as the number of virtual fields leading to a final system of the type of system is a priori infinite, thus leading to the search of some guidelines to help find optimal ones, meaning that a relevant criterion has to be chosen. In the present problem, an important feature is the fact that data processed are collected by a measurement device. They are therefore noisy and they lead to a bias in the determination of the parameters as the inversion of $\mathbf{P}$ for solving system 4.4 may magnify this noise and may lead to incorrectly identified parameters if $\mathbf{P}$ is ill-conditioned [19].

The effect of noise is related to the condition number of matrix $\mathbf{P}$ in Equation (4.4). It is the ratio of the largest singular value of $\mathbf{P}$ to the smallest [19]. The effect of noise on the inversion of matrix $\mathbf{P}$ is normally minimised if the condition number is equal to 1 . Such a property is obtained if matrix $\mathbf{P}$ in Equation (4.4) is equal to identity $(\mathbf{P}=\mathbf{I})$. This can be achieved with a relevant choice of the virtual fields which are called special virtual fields in this case.

\subsection{Empirically defined virtual fields}

As a first attempt, the virtual fields can be selected empirically, following some rules such as zeroing some of the integrals in Equation (4.3), and there- fore some components in matrix $\mathbf{P}$ involved in system 4.4. This approach is however not completely satisfactory as the determination of the virtual fields (which directly influence the stability of the solution found in case of noisy data) is somewhat arbitrary (see Grédiac and Vautrin [20] for instance). This has led the authors to find some guidelines to propose a more systematic construction of these virtual fields, as shown in the following section.

\subsection{Special virtual fields}

An orthotropic elastic law is considered herein for the sake of simplicity. The constitutive equation only depends on four parameters $Q_{11}, Q_{22}, Q_{12}$ and $Q_{66}$. In this case, Equation (4.3) can be written as follows:

$$
\begin{gathered}
Q_{11} \underbrace{\int_{V} \varepsilon_{1} \varepsilon_{1}^{*} \mathrm{~d} V}_{=1 \mathrm{~m}^{3}}+Q_{22} \underbrace{\int_{V} \varepsilon_{2} \varepsilon_{2}^{*} \mathrm{~d} V}_{=0 \mathrm{~m}^{3}}+Q_{12} \underbrace{\int_{V}\left(\varepsilon_{1} \varepsilon_{2}^{*}+\varepsilon_{2} \varepsilon_{1}^{*}\right) \mathrm{d} V}_{=0 \mathrm{~m}^{3}} \\
+Q_{66} \underbrace{\int_{V} \varepsilon_{6} \varepsilon_{6}^{*} \mathrm{~d} V}_{=0 \mathrm{~m}^{3}}=\int_{S_{f}} \overline{\mathbf{T}}(M, \mathbf{n}) \cdot \mathbf{u}^{*}(M) \mathrm{d} S .
\end{gathered}
$$

Constructing virtual fields leading matrix $\mathbf{P}$ to be equal to I means that they must be such that one of the integrals in Equation (4.5) is equal to 1 whereas the remaining ones are equal to zero, as shown below the brackets under each term of Equation (4.5). For instance, a first virtual field must be found so that the coefficient of $Q_{11}$ above is equal to one and the remaining ones are equal to zero. Three other virtual fields must be found so that the location of the ' 1 ' moves from one integral to another. With these four virtual fields, system 4.4 becomes

$$
\mathbf{Q}=\mathbf{R}
$$

as $\mathbf{P}=\mathbf{I}$. The unknown parameters are directly found if the special virtual fields are known. The question of the practical construction of the special virtual fields arises therefore at this stage. Two ways have been investigated by the authors in the recent past. A first idea is to expand the virtual displacement fields with a set of functions such as monomials for instance. In the case of an in-plane problem, one can write in this case:

$$
\left\{\begin{array}{l}
u_{1}^{*}=\sum_{i=0}^{m} \sum_{j=0}^{n} A_{i j} x^{i} y^{j} \\
u_{2}^{*}=\sum_{i=0}^{p} \sum_{j=0}^{q} B_{i j} x^{i} y^{j}
\end{array}\right.
$$


Coefficients $A_{i j}$ and $B_{i j}$ of these monomials fully define the special virtual fields. A second possibility is to define the virtual fields in a piecewise form, using an approach similar to the construction of the actual displacement field in the finite element method. This leads to 'virtual elements' defining the virtual fields completely from the virtual degrees of freedom (dofs) of the virtual nodes and appropriate shape functions. One can write

$\mathbf{u}^{*}=\mathbf{F U}^{*}$,

where $\mathbf{u}^{*}$ is a vector containing the virtual displacements $u_{1}^{*}$ and $u_{2}^{*}, \mathrm{~F}$ is a $2 \times p$ matrix containing the shape functions of the virtual element, $\mathbf{U}^{*}$ is a vector containing the virtual dofs which define the special virtual fields in this case. Its size depends on the nature of the shape functions and on the number of virtual elements.

Either the polynomial coefficients or the virtual nodal displacements are determined writing that the virtual field is special:

- one integral in Equation (4.5) is equal to one and the three remaining ones are equal to zero. This leads to a set of four linear equations where the coefficients or the virtual nodal displacements are unknown;

- the virtual field is kinematically admissible, meaning that the virtual displacements along $S_{u}$ are zero. This provides an additional set of linear equations the number of which depends on the problem.

Some additional conditions can be added. For instance, if measurements are not available on some part of the specimen, a virtual field which is different on both areas of the specimen (area where measurements are available and area where they are not) must be defined. The idea is to construct solid rigid like virtual fields on the second area. The virtual strain components are therefore zero in this case and the internal virtual work is consequently zero too, thus cancelling the contribution of this area in Equation (4.5). Such a situation is described in Grédiac et al. [21].

An important feature must be underlined at this stage. The number of equations used for the determination of the special virtual fields is limited whereas the number of unknowns is not as this latter only depends on $m, n, p$ and $q$ in Equation (4.7) (or on the number of virtual elements if piecewise virtual fields are used). The number of equations may therefore be less than the number of unknowns: special virtual fields are in fact not unique and an infinite number may be found. This extra freedom is used in the following section to minimise noise effect on identified values.

\subsection{Optimised special virtual fields}

Because of noisy data, the measured values of $\varepsilon_{1}, \varepsilon_{2}$ and $\varepsilon_{6}$ used for the identification are different from the actual ones. Some noise is added to them and the principle of virtual work is only rigorously verified provided that the noise is subtracted from the measured values. Hence the expression given in Equation (4.6) would rather be in reality:

$(\mathbf{I}-\mathbf{E}) \mathbf{Q}=\mathbf{R}$,

where $\mathbf{E}$ is the matrix giving the deviation from the theoretical equation due to error sources in the measurements. Therefore, the identified stiffness will actually be:

$\mathbf{Q}=\mathbf{R}+\mathbf{E} \mathbf{Q}$.

It is assumed in the following that error sources are random processes $[22,23]$. Thus, the identification error EQ is a random vector which can be analysed using statistics. Accordingly, its variance matrix can be written:

$\mathcal{V}(\mathbf{Q})=\mathcal{E}\left(\mathbf{E Q}^{\mathrm{t}} \mathbf{Q}^{\mathrm{t}} \mathbf{E}\right)$,

where $\mathcal{E}(\mathbf{X})$ denotes the mathematical expectation of a random vector $\mathbf{X}$ and $\mathrm{X}^{\mathrm{t}}$ denotes the transpose of vector $\mathbf{X} . \mathcal{V}(\mathbf{Q})$ is a matrix which contains along its diagonal the variances of each identified parameter $Q_{i j}$, i.e. indicators of uncertainty of the identification. The off diagonal terms are covariance coefficients which are useless here.

The optimisation of the virtual fields will aim at minimising the diagonal components of $\mathcal{V}(\mathbf{Q})$. An orthotropic elastic law is still considered herein for the sake of simplicity. Minimising $\mathcal{V}(\mathbf{Q})$ is used as the criterion for choosing the four special virtual fields $\mathbf{u}^{*[1]}, \mathbf{u}^{*[2]}, \mathbf{u}^{*[3]}$ and $\mathbf{u}^{*[4]}$ necessary to identify the respective four unknown parameters $Q_{11}, Q_{22}, Q_{12}$ and $Q_{66}$.

The random process which is used to model error sources in the measurements is merely an $\Re$-valued Gaussian white noise on $\Re^{3}$. It is assumed that three independent copies of this white noise add to each component of the actual strain fields in the measurements. In this case of error modelling, it was shown that the $\mathbf{u}^{*[k]}$ fields have to minimise the following functional to be optimal [23]: 


$$
\begin{aligned}
\mathcal{F}\left(\mathbf{u}^{*[k]}\right)= & \frac{1}{2}\left\{\left[Q_{11}^{2}+Q_{22}^{2}\right] \int_{V}\left[\varepsilon_{1}\left(\mathbf{u}^{*[k]}\right)\right]^{2} \mathrm{~d} V\right. \\
& +\left[Q_{22}^{2}+Q_{12}^{2}\right] \int_{V}\left[\varepsilon_{2}\left(\mathbf{u}^{*[k]}\right)\right]^{2} \mathrm{~d} V \\
& +Q_{66}^{2} \int_{V}\left[\varepsilon_{6}\left(\mathbf{u}^{*[k]}\right)\right]^{2} \mathrm{~d} V+2\left[Q_{12}\left(Q_{11}+Q_{22}\right)\right] \\
& \left.\times \int_{V}\left[\varepsilon_{1}\left(\mathbf{u}^{*[k]}\right) \varepsilon_{2}\left(\mathbf{u}^{*[k]}\right)\right] \mathrm{d} V\right\}
\end{aligned}
$$

A virtual field which verifies the condition to be special (this last property includes the kinematical admissibility) and which minimises the $\mathcal{F}$ cost function is unique. It is the solution of a constrained minimisation problem, where specialty is the constraint and $\mathcal{F}$ is the cost function.

The virtual fields are either polynomial functions, or piecewise defined continuous functions. The vectors composed either of the monomial coefficients or of the virtual nodal displacements are determined by solving the constrained minimisation problem, which can be written finally after deriving its Lagrangian [23]:

$$
\begin{aligned}
& {\left[\begin{array}{l|c}
{[\mathbf{H}} & \mathbf{A}^{\mathrm{t}} \\
\hline \mathbf{A} & 0
\end{array}\right]\left\{\begin{array}{cccc}
\mathbf{U}^{*[1]} & \mathbf{U}^{*[2]} & \mathbf{U}^{*[3]} & \mathbf{U}^{*[4]} \\
\boldsymbol{\Lambda}^{[1]} & \boldsymbol{\Lambda}^{[2]} & \boldsymbol{\Lambda}^{[3]} & \boldsymbol{\Lambda}^{[4]}
\end{array}\right\}} \\
& \quad=\left\{\begin{array}{cccc}
\mathbf{0} & \mathbf{0} & \mathbf{0} & \mathbf{0} \\
\mathbf{Z}^{[1]} & \mathbf{Z}^{[2]} & \mathbf{Z}^{[3]} & \mathbf{Z}^{[4]}
\end{array}\right\},
\end{aligned}
$$

where:

- $\mathbf{U}^{*[1]}, \mathbf{U}^{*[2]}, \mathbf{U}^{*[3]}$ and $\mathbf{U}^{*[4]}$ are the vectors containing either the monomial coefficients or the virtual nodal displacements of the four virtual fields necessary to identify respectively $Q_{11}$, $Q_{22}, Q_{12}$ and $Q_{66}$;

- $\quad \mathbf{A}$ is a matrix containing the linear constraints defined by the specialty of the virtual fields;

- $\quad \mathbf{H}$ is a matrix such that

$$
\mathcal{F}\left(\mathbf{u}^{*[k]}\right)=\frac{1}{2} \mathbf{U}^{*[k] t} \mathbf{H} \mathbf{U}^{*[k]}
$$

- $\quad \Lambda^{[1]}, \Lambda^{[2]}, \Lambda^{[3]}$ and $\Lambda^{[4]}$ are the Lagrange multipliers;

- $\quad \mathbf{Z}^{[1]}, \mathbf{Z}^{[2]}, \mathbf{Z}^{[3]}$ and $\mathbf{Z}^{[4]}$ are the vectors associated with the constraints. All their components are zero except one which is equal to 1 so as to provide special virtual fields;

- $\mathrm{t}$ is transposition.

Solving Equation (4.13) yields the four vectors $\mathbf{U}^{*[1]}, \mathbf{U}^{*[2]}, \mathbf{U}^{*[3]}$ and $\mathbf{U}^{*[4]}$ from which are deduced the four virtual fields $\mathbf{u}^{*[1]}, \mathbf{u}^{*[2]}, \mathbf{u}^{*[3]}$ and $\mathbf{u}^{*[4]}$ necessary to identify respectively $Q_{11}, Q_{22}, Q_{12}$ and $Q_{66}$. However, the problem becomes implicit because $Q_{11}, Q_{22}, Q_{12}$ and $Q_{66}$ are unknown at the beginning of the procedure and they are involved in the expression of $\mathcal{F}\left(\mathbf{u}^{*[k]}\right)$. This problem is solved by an iterative algorithm where the unknown parameters are replaced by their identified values at each iteration. Random values are input for the first iteration. Tests show that this algorithm converges in practice in less than four iterations whatever the choice of initial values for the unknown parameters [23].

\subsection{Optimised piecewise special virtual fields}

The objective here is to show how optimised special virtual fields based on the procedure described above can be obtained using piecewise functions to expand them. In this case, the virtual fields are defined by subdomains. The volume of the specimen $V$ is divided into $p$ subdomains such that $V=$ $V_{1} \cup V_{2} \cup \cdots V_{p}$. The left hand side integrals in Equation (4.5) can be written in this case:

$$
\left\{\begin{array}{l}
\int_{V} \varepsilon_{1} \varepsilon_{1}^{*} \mathrm{~d} V=\sum_{i=1}^{p} \int_{V_{i}} \varepsilon_{1} \varepsilon_{1}^{\star} \mathrm{d} V \\
\int_{V} \varepsilon_{2} \varepsilon_{2}^{*} \mathrm{~d} V=\sum_{i=1}^{p} \int_{V_{i}} \varepsilon_{2} \varepsilon_{2}^{\star} \mathrm{d} V \\
\int_{V}\left(\varepsilon_{1} \varepsilon_{2}^{*}+\varepsilon_{2} \varepsilon_{1}^{*}\right) \mathrm{d} V=\sum_{i=1}^{p} \int_{V_{i}}\left(\varepsilon_{1} \varepsilon_{2}^{\star}+\varepsilon_{2} \varepsilon_{1}^{\star}\right) \mathrm{d} V \\
\int_{V} \varepsilon_{6} \varepsilon_{6}^{*} \mathrm{~d} V=\sum_{i=1}^{p} \int_{V_{i}} \varepsilon_{6} \varepsilon_{6}^{\star} \mathrm{d} V
\end{array}\right.
$$

where $V_{i}$ is the volume of each subdomain. The shape of the subdomains is chosen in such a way that the virtual displacements $\mathbf{u}^{*}$ can be written as an expansion of shape functions $F_{i}$ multiplied by the nodal virtual displacements $u_{i}^{\star}$ as in the well-known finite element method:

$\mathbf{u}^{*}=\sum_{i=1}^{n_{\text {nodes }}} F_{i} u_{i}^{\star}=\mathbf{F} u^{\star}$,

where $n_{\text {nodes }}$ is the number of nodes per subdomain.

The virtual strain components in each subdomain are determined by differentiating Equation (4.15).

By introducing virtual strains in Equations (4.5) and (4.14), conditions between the virtual nodal displacements to get special virtual fields are obtained. These conditions lead to a linear system where $Y^{\star}$ is unknown

$\mathbf{A} Y^{\star}=\mathbf{B}$.

Vector $Y^{\star}$ is the vector of the degrees of freedom of all the virtual nodes of the virtual subdomains. Matrices $\mathbf{A}$ and $\mathbf{B}$ are obtained by assembling the subdomains used to mesh surface $S$. It should be emphasised that 
the dimension of unknown vector $Y^{*}$ is $k N \times 1$ where $N$ is the number of virtual nodes and $k$ is the number of degrees of freedom for each node. The number of equations available is the sum of the $s$ special virtual fields conditions (four in the present approach for an orthotropic material) and of $b$ boundary conditions. If $s+b$ is smaller than the number of unknown degrees of freedom $N$, matrix $P$ becomes rectangular and one has to add some other conditions to find the virtual nodal displacements. These additional conditions can be arbitrary or may arise from some additional requirements, like the minimisation of the effect of noise on the identified data [23] described above.

Piecewise virtual fields present several advantages over a continuous expansion like polynomials. Among them, the degree of the shape functions is generally much lower than that of continuous polynomials, thus reducing the effect of noise carried on to the final results. The use of shape functions gives more freedom to construct either one or many piecewise virtual fields over the whole geometry of the specimen. This is of particular importance for configurations using complex shapes or containing discontinuities (holes, cracks, etc.). A third advantage is that displacement virtual fields have to respect the $C^{0}$ continuity only and can therefore allow $C^{1}$ discontinuities between subdomains without introducing any error in the nodal virtual displacement calculations, and therefore in the identification of the constitutive parameters.

\section{Elastic Bending Stiffness of Thin Anisotropic Plates}

From an historical point of view, the problem of plate bending is the first one to be addressed with the method. In this case, equations defined above slightly differ as the assumption of a constant through-thickness strain distribution becomes irrelevant. The Love-Kirchhoff assumption describes the strain distribution in this case [24]. The unknown parameters are the bending stiffnesses which link bending moments to curvatures

$\left[\begin{array}{l}M_{1} \\ M_{2} \\ M_{6}\end{array}\right]=\left[\begin{array}{lll}D_{11} & D_{12} & D_{16} \\ D_{12} & D_{22} & D_{26} \\ D_{16} & D_{26} & D_{66}\end{array}\right]\left[\begin{array}{l}k_{1} \\ k_{2} \\ k_{6}\end{array}\right]$,

where the $D_{i j} \mathrm{~s}$ are the bending stiffnesses, the $M_{i} \mathrm{~s}$ are the generalised bending moments and the $k_{i}$ s are the curvatures defined by:

$k_{1}=-\frac{\partial^{2} u_{3}}{\partial x_{1}^{2}} ; \quad k_{2}=-\frac{\partial^{2} u_{3}}{\partial x_{2}^{2}} ; \quad k_{6}=-2 \frac{\partial^{2} u_{3}}{\partial x_{1} \partial x_{2}}$ where $u_{3}$ is the out-of-plane displacement component. After some developments which are not recalled here (full details may be found in Grédiac and Vautrin [20] or Grédiac et al. [25]), the principle of virtual work may be written as follows:

$$
\begin{aligned}
& D_{11} \int_{S} k_{1} k_{1}^{*} \mathrm{~d} S+D_{22} \int_{S} k_{2} k_{2}^{*} \mathrm{~d} S+D_{12} \int_{S}\left(k_{1} k_{2}^{*}+k_{2} k_{1}^{*}\right) \mathrm{d} S \\
& \quad+D_{66} \int_{S} k_{6} k_{6}^{*} \mathrm{~d} S+D_{16} \int_{S}\left(k_{1} k_{6}^{*}+k_{6} k_{1}^{*}\right) \mathrm{d} S \\
& \quad+D_{26} \int_{S}\left(k_{2} k_{6}^{*}+k_{6} k_{2}^{*}\right) \mathrm{d} S=W_{e}^{*}
\end{aligned}
$$

In Grédiac and Vautrin [20, 26] for instance, virtual deflection fields $u_{3}^{*}$ are defined empirically whereas they are special in Grédiac et al. [27]. Actual curvature fields can be obtained in practice by differentiation of slope fields measured with a deflectometry setup [28] or by double differentiation of the deflection fields [29]. In Grédiac [25] and Grédiac et al. [30], virtual fields are chosen empirically and invariant parameters governing the anisotropic bending law are extracted. In Grédiac and Paris [31] and Grédiac et al. [32], the loading is no longer static but dynamic. This requires the inertial term (third term in Equation 3.1) to be taken into account.

\section{In-Plane Anisotropic Stiffnesses}

\section{I. Overview}

Soon after the initial study on bent plates, the first applications of the VFM to the in-plane anisotropic stiffness components of composite plates were launched. The very first attempt focused on a standard double notched shear specimen taken from an American Society for the Testing of Materials (ASTM) standard, also called the Iosipescu specimen [33]. The idea was to determine the in-plane shear modulus by relating the average shear stress to the average shear strain derived from full-field measurements [34] and attempts were also made at using displacements at the boundary of the gauge section instead of the average shear strain over the surface, which did not prove so successful from a practical point of view. Soon after that, a T-shaped specimen was specifically designed to try to balance the contribution of the different stress components to optimise identifiability. This was performed through topological optimisation and a criterion on the stress components [35]. The experimental implementation was reasonably successful but the quality of the full-field measurements was not sufficient to 
provide very good results [36]. Several other configurations were then examined, mainly using simulated data, among which the three-point bending test on thick specimens [37] which again did not prove very successful from an experimental point of view.

The two main configurations that have been more extensively studied by the authors are now reported in more detail. But first, a short description of the full-field measurement technique used in most of the cases presented in the following is given.

\subsection{Full-field measurements with the grid method}

The displacement fields required for the model identification are measured using an optical method called the grid method [38-40]. A grid pattern is deposited onto the surface of the specimen [41]. The grid period is $100 \mu \mathrm{m}$. Images of the undeformed and deformed gratings are digitised through a $1280 \times$ 1024 CCD camera connected to a PC. The objective of the camera is set such that a period of the grid is sampled by approximately 4 pixels.

\subsection{Printing of the grid}

The spatial carrier consists in the superposition of horizontal and vertical black lines printed over a white surface, with a natural spatial frequency $f_{0}=10$ lines per $\mathrm{mm}$. This has been achieved by applying the procedure detailed in Piro and Grédiac [41].

\subsubsection{Characterisation of the} displacements by a phase modulation

At any state, the digitised light at a given pixel $M_{0}$ is the light reflected by a material point $M$ determined by its position $(X, Y)$ in the reference Cartesian frame $(\mathrm{O}, \mathbf{i}, \mathbf{j})$. Its intensity can be written:

$$
I(X, Y)=I_{0}\{1+\gamma \text { frng }[2 \pi X \mathbf{F} . \mathbf{i}+2 \pi Y \mathbf{F} . \mathbf{j}]\}
$$

where:

- $I_{0}$ is the local intensity bias,

- $\gamma$ is the contrast,

- frng is a $2 \pi$-periodic continuous function, which is normally a sinusoid possibly with some higher harmonics at relatively low amplitude,

- $\because$ denotes the dot product,

- $2 \pi \mathbf{F} . \mathbf{R}$ is called the phase of function frng,

- $\quad \mathbf{F}$ is the spatial frequency vector. It is orthogonal to the grid lines and its amplitude is the spatial frequency of the grid. If the grid lines are vertical, they are parallel to $\mathbf{j}$, meaning that the spatial frequency vector can be written as $\mathbf{F}=\left(f_{0}, 0\right)$. If the grid lines are horizontal, they are parallel to $\mathbf{i}$, meaning that the spatial frequency vector can be written as $\mathbf{F}=\left(0, f_{0}\right)$.

When a load is applied, there is a deformation of the structure and the grid is also deformed. From the undeformed to the deformed state, the phase of the function 'frng' at pixel $M_{0}$ varies as:

$\Delta \phi=-2 \pi u_{1}(X, Y) \mathbf{F} . \mathbf{i}-2 \pi u_{2}(X, Y) \mathbf{F} . \mathbf{j}$,

where $\mathbf{u}(X, Y)$ is the displacement vector at $(X, Y)$. Accordingly, the first component $u_{1}(X, Y)$ of the displacement is calculated from the phase of the 'frng' function for the vertical lines, the second component $u_{2}(X, Y)$ for the horizontal lines. The phase fields are computed by using the spatial phase shifting method implemented in a Matlab routine $[38,42]$.

\subsubsection{Measurement of the displacement fields}

The $u_{1}(X, Y)$ and $u_{2}(X, Y)$ displacement components relative to the unloaded reference condition are calculated from the respective phase differences $\Delta \phi_{1}$ (for vertical lines) and $\Delta \phi_{2}$ (for horizontal lines) introduced by the deformation:

$$
\begin{aligned}
& u_{1}(X, Y)=-\frac{p}{2 \pi} \Delta \phi_{1}(X, Y), \\
& u_{2}(X, Y)=-\frac{p}{2 \pi} \Delta \phi_{2}(X, Y),
\end{aligned}
$$

where $p=0.1 \mathrm{~mm}$. The resolution of the method, i.e. the smallest displacement which can be measured in absolute value, depends on the measurement noise. The noise is a random variable which adds to the phase during the calculation [42]. Its mean is 0 and its standard deviation is denoted $\sigma_{\phi}$.

Of the current experiments, $\sigma_{\phi}$ was at most $2 \pi / 100$, meaning that the resolution of the method for measuring displacements was at worst $1 \mu \mathrm{m}$. This was evaluated by taking two images of the undeformed grid and calculating the standard deviation of the detected phase. The spatial resolution, i.e. the smallest distance which separates two independent displacement values, equaled two periods $=$ $0.2 \mathrm{~mm}=8$ pixels.

\subsubsection{Computation of strain fields}

In practice, it is difficult to get strain fields with a sharp spatial resolution because measured displacement fields are noisy due to the incertitude of optical set-ups. 
Noise can be processed using filtering. Several approaches are available. One of the most famous consists of applying a square convolution kernel over the image for smoothing it. However, this technique can fail with experimental data near the edges of the specimen or around small area where data are missing or significantly altered. In these areas, local defects in the measurements are widespread but not eliminated. It is therefore rather improbable that the principle of virtual work can be applied to such derived strain fields. This is why strain fields obtained with this filtering approach from experimental displacement fields, and then processed with the VFM, can yield aberrant values for stiffness parameters as was the case in [43] for instance.

An alternative approach consists of fitting the data with 2D polynomial functions by least square minimisation [44]. It is possible to compute the polynomial function by minimising the deviation from measured displacements only at locations where there are no defects using a weighted least square approach. Finally, missing data are interpolated with the computed polynomial functions. Such an algorithm was implemented in Matlab for fitting the measured displacement fields.

\subsection{Thick composite rings}

This application concerns the measurement of the through-thickness stiffness components of thick composite rings cut from thick tubes obtained from filament winding. This type of structure is used for underwater applications (casing for electronic measurement devices, for instance). The tubes undergo very high external pressure loads and must therefore be thick enough to withstand these loads. In this case however, through-thickness stiffness components are required to calculate the response of the tube and because of the cylindrical symmetry of the tube, standard tests based on rectangular coupons are extremely difficult to perform.

The alternative is to use a simple diametral compression test, measure full-field strains and use the VFM. A first theoretical and numerical study was released a few years ago [45] and the experimental implementation has just been completed very recently. The mechanical set-up is shown in Figure 2. Two back-to-back cameras have been used in order to take into account both the out-of-plane movements that create parasitic hydrostatic strains because of the change in magnification caused by the lens and the possible non-uniform strain distribution due to the fixture misalignment. This has proved absolutely necessary, as was demonstrated in [46]. All

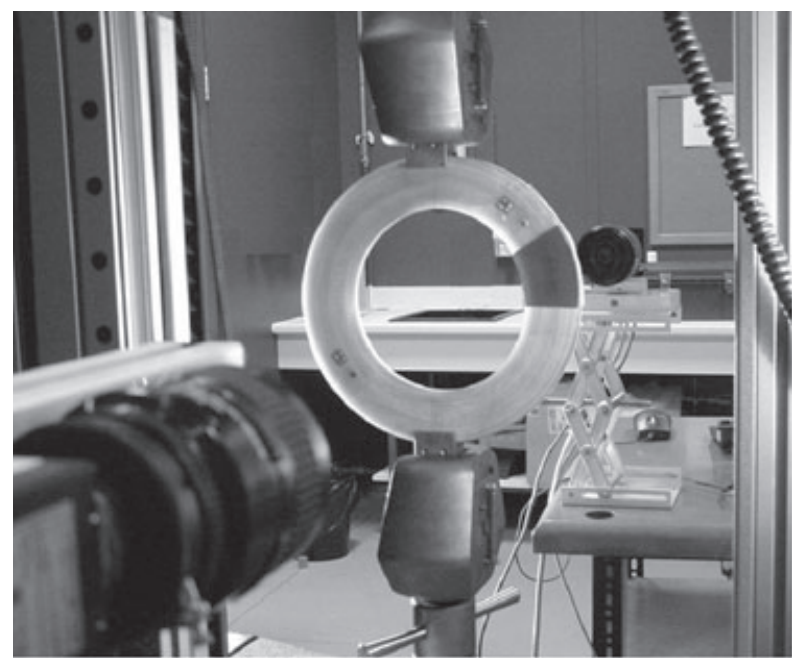

Figure 2: Thick composite ring in compression with the two cameras set

displacement maps have therefore been obtained by averaging the back-to-back data.

The displacement field was measured over a specific area of the ring with the grid method (see Figure 2). If the whole ring had been considered, a lot of pixels of the camera would have been lost because of the cylindrical geometry, hence the arrangement shown in Figure 2. However, the position of the instrumented area has been selected in order to optimise the identifiability of the different stiffness components [46]. The displacement maps have been fitted by a 4 th order polynomial and the strains derived from the expression of the polynomials. Typical strain maps are given in Figure 3.

The same hoop-wound glass-epoxy ring has been tested nine times and between two consecutive tests, the ring has been taken out of the rig and put back again. The strain fields have been processed using piecewise optimised virtual fields. The results are reported in Table 1.

The results are very satisfactory when compared with reference data. In particular, the coefficients of variation exhibit the same trend as the coefficients of sensitivity to noise that are output from the VFM with noise optimisation. They can be considered as a confidence index for the different components identified. It must also be pointed out that the reference values are only generic data for a unidirectional glass-epoxy composite as no reference test can be undertaken for this configuration. The rather high shear modulus is rather surprising but might be cause by very high fibre volume fraction. In any case, the procedure has proved very powerful to obtain stiffness data otherwise extremely difficult to measure. 
(A)

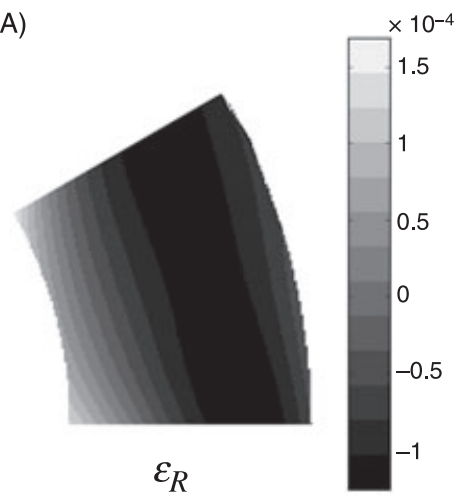

(B)

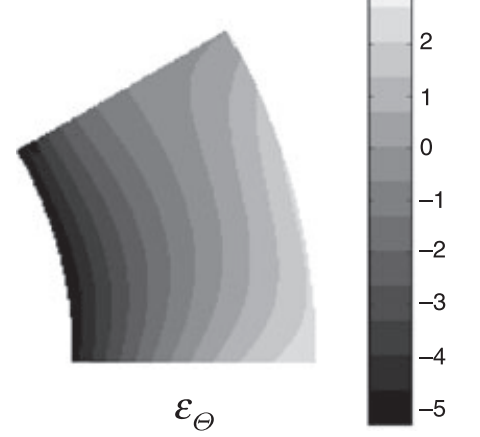

(C)

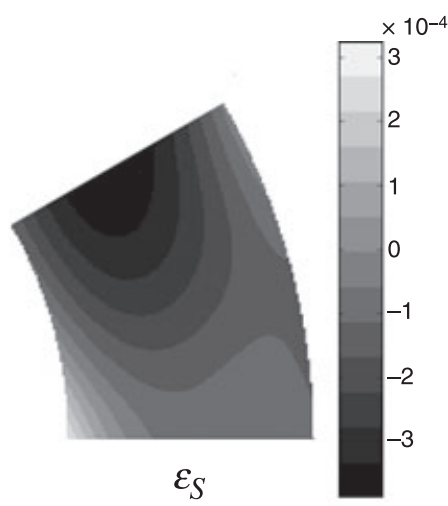

(D)

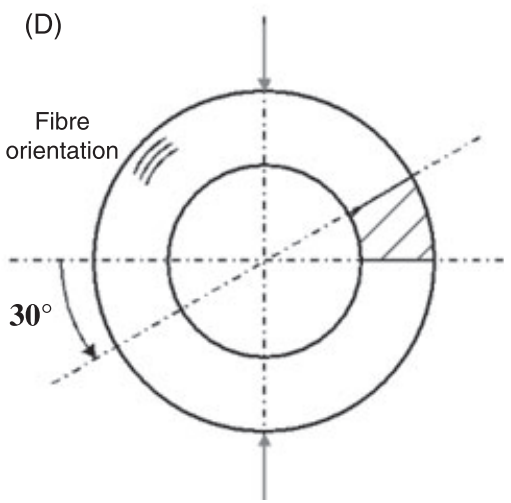

Figure 3: Experimental strain fields $(F=20 \mathrm{kN})$

Table I: Identified rigidities of the hoop-wound glass-epoxy ring using the improved set-up with two cameras

\begin{tabular}{lclcl}
\hline Test no. & $\begin{array}{l}Q_{R R} \\
(\mathrm{GPa})\end{array}$ & $\begin{array}{l}Q_{\Theta \Theta} \\
(\mathrm{GPa})\end{array}$ & $\begin{array}{l}Q_{R \Theta} \\
(\mathrm{GPa})\end{array}$ & $\begin{array}{l}Q_{S s} \\
(\mathrm{GPa})\end{array}$ \\
\hline 1 & 13.1 & 48.3 & 3.08 & 6.77 \\
2 & 12.6 & 45.4 & 2.04 & 6.93 \\
3 & 15.7 & 46.0 & 2.26 & 6.88 \\
4 & 13.3 & 44.6 & 1.92 & 6.36 \\
5 & 9.46 & 49.4 & 3.8 & 6.96 \\
6 & 10.9 & 47.2 & 3.86 & 6.41 \\
7 & 10.1 & 45.9 & 2.44 & 7.1 \\
8 & 8.44 & 39.6 & 2.2 & 6.54 \\
9 & 8.95 & 41.9 & 2.03 & 7.12 \\
Average & 11.4 & 45.4 & 2.62 & 6.78 \\
Coefficient of variation & $29 \%$ & $10 \%$ & $29 \%$ & $4 \%$ \\
Engineering constants & $E_{R R}$ & $E_{\Theta \Theta}$ & $v_{\Theta R}$ & $G_{R \Theta}$ \\
& $11.3 \mathrm{GPa}$ & $44.8 \mathrm{GPa}$ & 0.23 & $6.78 \mathrm{GPa}$
\end{tabular}

\subsection{Shear-bending test on rectangular coupons}

Another configuration extensively studied by the authors is the bending-shear loading of a rectangular coupon. This test is derived from the Iosipescu fixture (ASTM standard with a double V-notched specimen [33]) that is used to measure the shear modulus of orthotropic composites. Following an initial study mentioned previously [34], it was devised that by removing the notches on the specimen, a heterogeneous stress/strain field can be obtained that may enable the simultaneous identification of the four in-plane stiffness component. A first feasibility study based on simulated data and using empirical virtual fields confirmed this opinion [47].

This test consists of loading a straight rectangular beam using the Iosipescu fixture (Figure 4). The $\mathrm{V}$-notches in the classical geometry of the Iosipescu specimen [33] were removed and the length between the supports was increased [21, 43, 48]. Optimised piecewise virtual field were used here. The virtual mesh defined over the specimen is divided into three areas: S1, S2 and S3 (Figure 4).

The virtual displacement field was imposed as rigid body like over S1 and S3. Regarding S2, the area of interest, it is meshed with $3 \times 7$ rectangles. Thus, it is composed of 32 virtual nodes.

The four nodes located on the left side of S2 are constrained to have a zero displacement value so as to ensure continuity with $\mathrm{S} 1$. The horizontal displacement of the nodes located on the right hand side of S2 was also fixed to zero and the vertical displacements were constrained to be constant over the whole height of this edge, ensuring continuity between S2 and S3. An example of obtained virtual fields is given in Figure 5. 

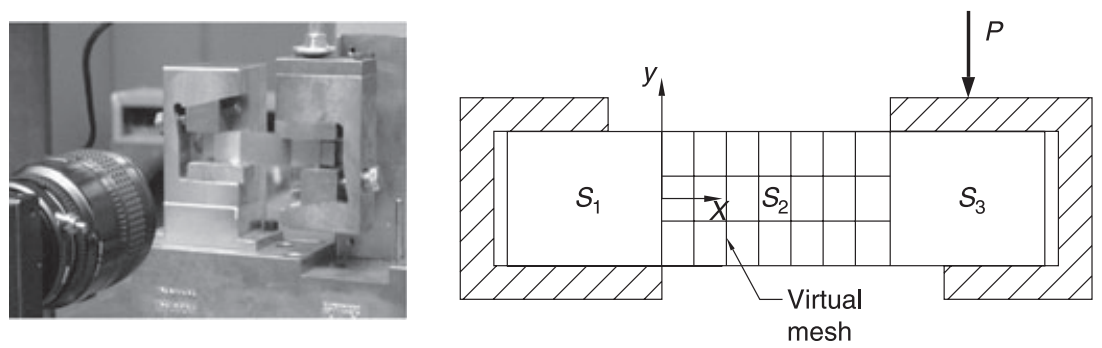

Figure 4: Bending/shear test based on the Iosipescu fixture
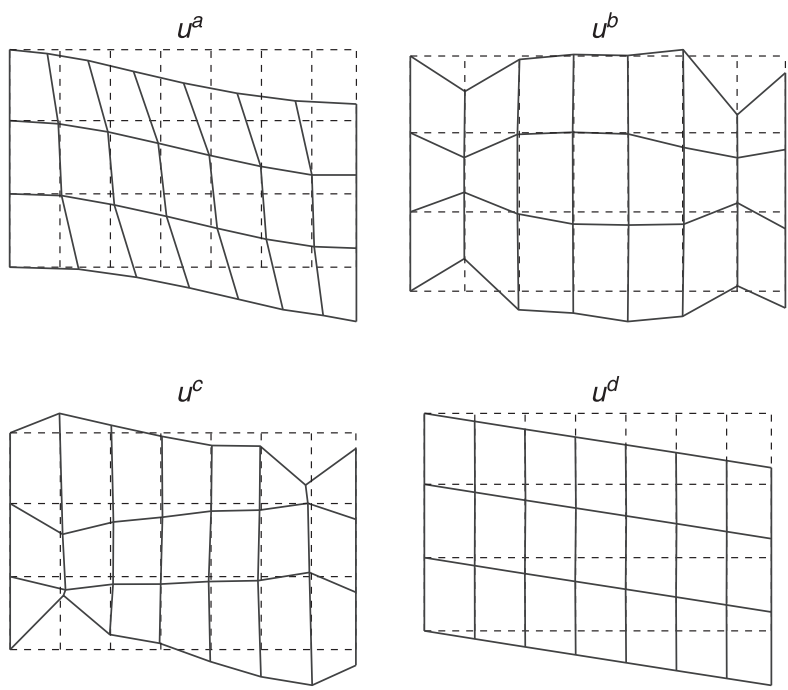

Figure 5: Optimised piecewise virtual fields used for the identification of stiffness parameters
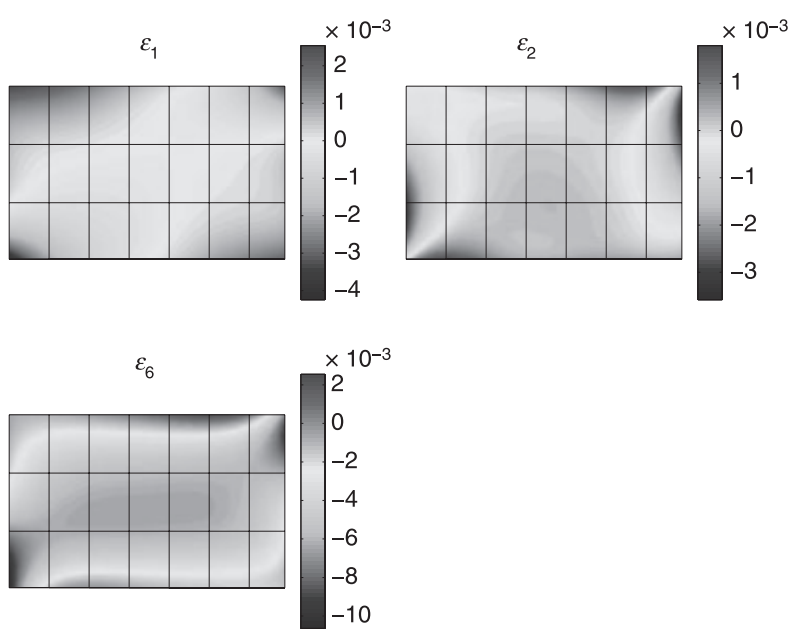

Figure 6: Strain fields computed from measured displacement fields (load: $700 \mathrm{~N}$ )

The displacement maps have been fitted by 7th order polynomials which were then used to compute the strain maps. Typical strain maps have been plotted in Figure 6. The identification results are reported in Table 2 . They have been obtained using four unidirectional glass-epoxy specimens with loads up to a given value (here, $700 \mathrm{~N}$ ) so that the shear response remains linear $[43,48]$.
Table 2: Identified parameters with the Iosipescu fixture

\begin{tabular}{lrrcl}
\hline & $Q_{11}$ & $Q_{22}$ & $Q_{12}$ & $Q_{66}$ \\
\hline Standard tests & & & & \\
$\quad$ Average (GPa) & 44.9 & 12.2 & 3.86 & 3.68 \\
$\quad$ Coefficient of variation (\%) & 0.7 & 2.8 & 2.4 & 8.6 \\
$\begin{array}{l}\text { Virtual fields method } \\
\quad \text { Average (GPa) }\end{array}$ & 39.7 & 10.4 & 3.03 & 3.65 \\
$\quad$ Coefficient of variation (\%) & 6.6 & 23.2 & 13.1 & 2.41 \\
\hline
\end{tabular}

The results are very satisfactory, considering that the panel used for the reference test was not the same as that for the VFM tests. It has been checked that the coefficients of variation are consistent with the noise amplification factors from the VFM, thus giving a confidence index for the different values identified. This application is certainly ripe for industrial transfer.

\section{Extension to Non-Linear Shear Behaviour of Composites}

It is well known that unidirectional polymer matrix composites exhibit a significant non-linear behaviour in shear. The objective here is to extend the approach presented in the previous section to take into account this material non-linearity in shear. First attempts at this problem using empirical virtual fields [50] and then, polynomial special fields [51] have been released using simulated data. The experimental application is more recent $[43,48]$. There, the nonlinear response in shear has been modelled using the model developed in [51] that writes the shear stressstrain relationship as:

$\sigma_{6}=Q_{66}^{0} \varepsilon_{6}-K \varepsilon_{6}\left\langle\varepsilon_{6}-\varepsilon_{6}^{0}\right\rangle^{+}$

where $K$ and $\varepsilon_{6}^{0}$ are the parameters driving the model; $\varepsilon_{6}^{0}$ is the threshold from which the non-linearity initiates; $\langle X\rangle^{+}$means $X$ when $X \geq 0$, 0 otherwise.

Considering that the three other elastic stiffness components are already known from the approach of 
Table 3: Identified parameters with the Iosipescu fixture

\begin{tabular}{lllc}
\hline & $Q_{66}^{0}$ & $K$ & $\varepsilon_{6}^{0}$ \\
\hline $\begin{array}{l}\text { Standard tests } \\
\quad \text { Average } \\
\quad \text { Coefficient of variation (\%) }\end{array}$ & 8.6 & & \\
$\begin{array}{l}\text { Virtual fields method } \\
\text { Average }\end{array}$ & $3.65 \mathrm{GPa}$ & $87.7 \mathrm{GPa}$ & 0.004 \\
Coefficient of variation (\%) & 2.41 & 8.43 & 14.2 \\
\hline
\end{tabular}

Section 6.4, only a pure shear virtual field is considered (see the fourth one in Figure 5) to process the data and retrieve the parameters driving the shear model.

Feeding Equation (4.5) with the non-linear model and the pure shear virtual field, only $Q_{66}^{0}, \varepsilon_{6}^{0}$ and $K$ are involved in the principle of virtual work. Accordingly, $K$ and $\varepsilon_{6}^{0}$ should satisfy the following equality [43]:

$\mathcal{C}\left(K, \varepsilon_{6}^{0}\right)=Q_{66}^{0} \int_{S 2} \varepsilon_{6} \mathrm{~d} S-K \int_{S 2} \varepsilon_{6}\left\langle\varepsilon_{6}-\varepsilon_{6}^{0}\right\rangle^{+} \mathrm{d} S+\frac{P L}{e}=0$.

However, this equation does not yield a linear system of equations here. Moreover, Equation (7.2) is not fulfilled in practice because the model considered here is only an approximation of the actual nonlinear behaviour. Therefore, the identification of $\varepsilon_{6}^{0}$ and $K$ is achieved by minimising the sum of the $\left[\mathcal{C}\left(K, \varepsilon_{6}^{0}\right)\right]^{2}$ computed for all the load steps, using the least square method.

Four specimens were loaded up to $2000 \mathrm{~N}$ using the set-up described in Section 6.4. Displacement fields were measured every $25 \mathrm{~N}$ and strain fields were derived using polynomial fitting (Figure 6). Stiffness parameters were identified for a load of $700 \mathrm{~N}$ (see Section 6.4) and the non-linear parameters were identified using all the strain maps. Identified values of the non-linear model are reported in Table 3.

These results are very satisfactory and confirm the feasibility of the VFM to identify non-linear models. However, the authors are fully aware that this is only a first approach and that more work is needed to address more complex models taking into account the unloading, the dissipation, the permanent strains, etc. This remains very much an open problem for future research.

\section{Application to Damping Measurement of Thin Plates}

As mentioned in Section 5, a first successful application of the VFM in dynamics concerned the identi-

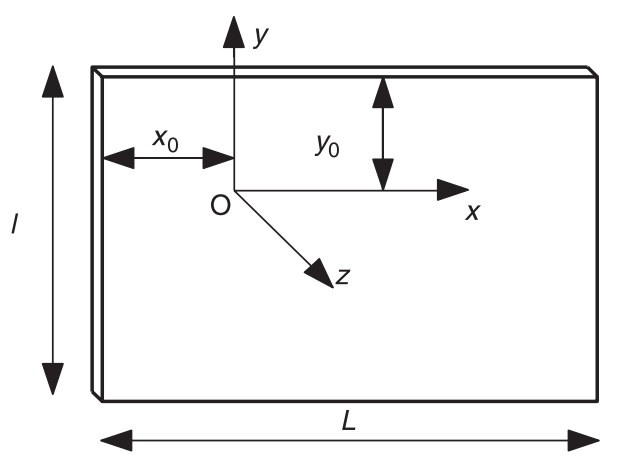

Figure 7: Rectangular plate under study

fication of anisotropic stiffness components of thin composite plates $[31,32]$. This work was recently extended to the measurement of material damping from vibration of thin plates.

\section{I. Theoretical summary}

Let us consider a rectangular thin plate. Its thickness is $h$ (Figure 7). The plate is clamped at point $O$ as seen in Figure 7, which is the origin of the coordinate system. Let us now suppose that this point is translated along the $z$-axis sinusoidally with time (inertial excitation). This arrangement is an extension of the experimental study of vibrating plates reported in Giraudeau et al. [52].

The actual strain and stress field across the plate are considered harmonic at the same frequency as the excitation movement (linearity assumption). The VFM is applied using complex virtual fields (i.e. harmonic virtual fields, at the same frequency as the excitation movement). Using a very simple damping model, it may be assumed that the generalised bending moment can be decomposed into an elastic bending moment $M^{\mathrm{e}}$ and a dissipative bending moment $M^{\mathrm{d}}$, with

$$
\left\{M^{\mathrm{e}}\right\}=\left[\begin{array}{l}
M_{1}^{\mathrm{e}} \\
M_{2}^{\mathrm{e}} \\
M_{6}^{\mathrm{e}}
\end{array}\right]=\left[\begin{array}{lll}
D_{11} & D_{12} & 0 \\
D_{12} & D_{11} & 0 \\
0 & 0 & \left(D_{11}-D_{12}\right) / 2
\end{array}\right] \cdot\left[\begin{array}{l}
k_{1} \\
k_{2} \\
k_{6}
\end{array}\right],
$$

which is the same as Equation (5.1) but for an isotropic material and

$$
\left\{M^{\mathrm{d}}\right\}=\left[\begin{array}{l}
M_{1}^{\mathrm{d}} \\
M_{2}^{\mathrm{d}} \\
M_{6}^{\mathrm{d}}
\end{array}\right]=\mathrm{j} \omega \cdot\left[\begin{array}{ccc}
B_{11} & B_{12} & 0 \\
B_{12} & B_{11} & 0 \\
0 & 0 & \left(B_{11}-B_{12}\right) / 2
\end{array}\right] \cdot\left[\begin{array}{l}
k_{1} \\
k_{2} \\
k_{6}
\end{array}\right]
$$

with $D$ the stiffness matrix, $B$ the damping matrix, $\mathrm{j}$ defined by $\mathrm{j}^{2}=-1, \omega$ the excitation pulsation and $k_{i}$ the components of the curvature field. Feeding this 
into the principle of virtual work in dynamics (neglecting the volume forces), a final system is obtained that can be written as:

$$
\begin{aligned}
D_{11} G_{r, r} & +D_{12} H_{r, r}-B_{11} \omega G_{i, r}-B_{12} \omega H_{i, r} \\
& =-\rho h \omega^{2} \int_{S}\left(d+w_{r}\right) w_{r}^{*} \mathrm{~d} S, \\
D_{11} G_{i, i} & +D_{12} H_{i, i}+B_{11} \omega G_{r, i}+B_{12} \omega H_{r, i} \\
& =-\rho h \omega^{2} \int_{S} w_{i} w_{i}^{*} \mathrm{~d} S,
\end{aligned}
$$

where the $G$ and $H$ functions can be written as:

$$
\begin{aligned}
& G_{p, q}=-\int_{S}\left(k_{1} k_{1}^{*}+k_{2} k_{2}^{*}+\frac{1}{2} k_{6} k_{6}^{*}\right) \mathrm{d} S \\
& H_{p, q}=-\int_{S}\left(k_{1} k_{2}^{*}+k_{2} k_{1}^{*}-\frac{1}{2} k_{6} k_{6}^{*}\right) \mathrm{d} S
\end{aligned}
$$

where $p$ and $q$ indicate that the $G_{p, q}$ and $H_{p, q}$ quantities are calculated using:

- the real or imaginary parts of the actual curvatures according to $p$ is $r$ or $i$;

- the real or imaginary parts of the virtual curvatures according to $q$ is $r$ or $i$.

This approach has been validated using finite element simulated data [53, 54]. A very interesting feature of the method is that it is not sensitive to damping coming from the clamping area. Also, resonance is not compulsory as the whole approach has been developed with a real representation rather than a frequency representation. So not only does it provide material damping (and not modal damping) but it is possible to sweep frequencies regardless of resonance, which confers to this approach a great potential for exploring the frequency dependance of the damping behaviour.

\subsection{Experimental implementation}

The experimental set-up is shown in Figure 8. The excitation is applied by an electrodynamic shaker connected to a metal rod itself connected to a jig that has the objective of guiding the movement and introducing some stiffness. The plate is bolted at the end of the connection rod so that a sinusoidal out-of-plane movement is applied to the plate. At the other end stands a wood panel on which a cross-line grating has been deposited ( $2 \mathrm{~mm}$ pitch). The tested plate must have a very smooth and reflective surface so that an image of the reflection of the grid can be formed. This image is captured by a CCD camera looking at the plate through a hole in the wood panel. When the plate deforms, the

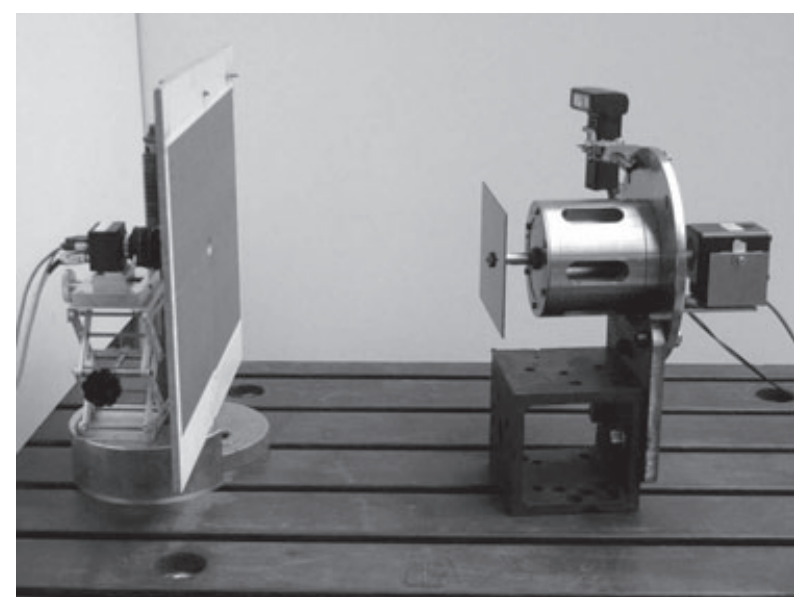

Figure 8: Experimental set-up for inertial excitation and deflectometry

local image of the grid is shifted according to the slope at the considered point. Therefore, this technique, often called deflectometry, can give direct access to slope fields in the two directions of the grid $[28,55]$. An accelerometer placed at the end of the rod gives the magnitude of the driving movement. In order to capture images at given times, a flash light is synchronised with the excitation and triggered to form the images. Therefore, both in-phase and out-of-phase slope maps can be captured.

Typical slope maps are given in Figure 9, together with the deflection field obtained by numerical integration, for a polycarbonate plate tested at $80 \mathrm{~Hz}$ [56]. Numerical differentiation is also performed to obtain the curvature fields.

Everything is then fed into Equations (8.3) and (8.4) using uniform curvature virtual fields [56]. First results for both Young's modulus, Poisson's ratio and damping factors are reported in Table 4. The damping factors are defined as:

$\beta_{11}=\frac{B_{11}}{D_{11}} \beta_{12}=\frac{B_{12}}{D_{12}}$

The reference values for $E$ has been obtained by cantilever beam vibration, as well as the damping parameter $\beta_{11}$, using logarithmic decrement of the vibration amplitude. A single beam has been tested four times, and for each test, the beam was taken out of the clamp and clamped again. For the plate, the same methodology was applied (same plate, four independent tests).

These first results are rather satisfactory but a lot of work is still needed to take advantage of the full potential of the very novel technique, in particular for anisotropic plates. 

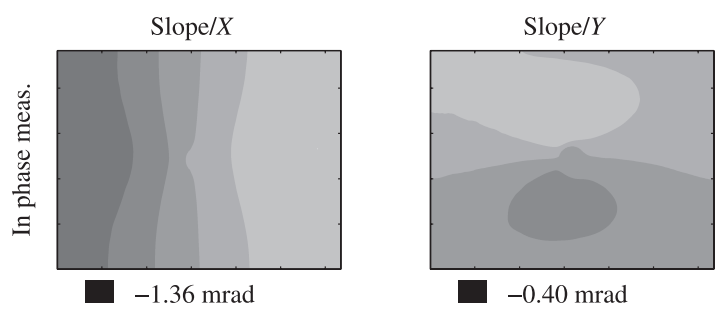

$-0.40 \mathrm{mrad}$

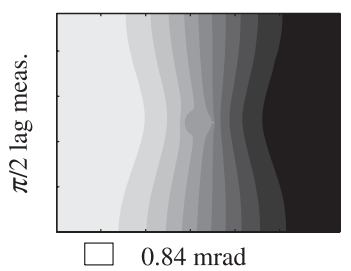

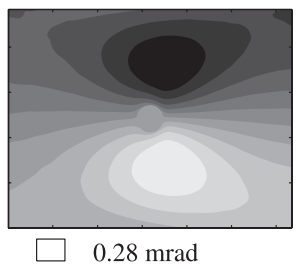

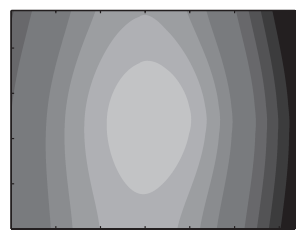

$0.15 \mathrm{~mm}$

Figure 9: Experimental in-phase and $\pi / 2$ lag slope and deflection fields in polycarbonate at $80 \mathrm{~Hz}$

Table 4: Identified parameters from test at $80 \mathrm{~Hz}$, polycarbonate plate

\begin{tabular}{llll}
\hline & $E(\mathrm{GPa})$ & $v$ & $\beta(\mathrm{s})$ \\
\hline Reference & 4.90 & - & $1.08 \times 10^{-4}$ \\
Coefficient of variation (\%) & 2.3 & - & 3 \\
Virtual fields method & 4.71 & 0.30 & $1.08 \times 10^{-4}$ \\
Coefficient of variation (\%) & 0.1 & 0.2 & 1.9 \\
\hline
\end{tabular}

\section{Application to Elasto-Plastic Behaviour}

\section{I. Introduction}

The identification of elasto-plastic constitutive equations is usually performed using statically determined tests such as tension or bending on a rectangular bar, torsion on a thin tube, etc. associated with local strain measurements (extensometers, dial gauges or LVDT transducers, strain gauges, etc.). The development of optical full-field strain measurements has opened the way for novel methodologies based on statically undetermined tests. Most of the time, the identification strategies rely on finite element model updating as explained in Section 2.2.

The VFM can be used as an alternative strategy to extract the parameters of an elasto-plastic constitutive equation on a complex test geometry when fullfield strain data are available. Its main advantage is that it does not require the use of finite element calculations and therefore, avoids the problem of boundary condition and specimen geometry modelling. In the following sections, the main features of the VFM applied to elasto-plasticity are recalled [57]. Furthermore an experimental validation on a simple application is presented [58].

\subsection{Theory}

With elasto-plastic constitutive equations, it is not possible to derive linear equations involving the unknown parameters as in Equation (4.3). If $X$ denotes the vector of unknown plastic constitutive parameters, the relationship between strains and stresses is a non-linear function which can be written as:

$\sigma(X, t)=Q\left[\varepsilon(t)-\varepsilon^{\mathrm{p}}(X, t)\right]$,

where ${ }_{\varepsilon}^{\mathrm{p}}$ is the plastic strain tensor, depending on time and involving the constitutive parameters. $\varepsilon(t)$ is the measured total strain tensor at time $t$ and $Q$ is the stiffness matrix. It is assumed that the stiffness matrix is constant all along the test. For identifying $X$ with the VFM, an iterative procedure is required because the stress tensor only depends implicitly on the unknown constitutive parameters through the evolution of plastic strains. The full test is divided in $m$ load steps denoted $t_{i}(i=1, \ldots, m)$ when strain fields are measured. A cost function to minimise is defined as follows:

- at each load step $t_{i}$, the field of plastic strain ${ }_{\varepsilon}^{\mathrm{p}}\left(X, t_{i}\right)$ is computed iteratively within the framework of elasto-plasticity, using the plastic strains computed at the previous steps and the measured total strain fields $[57,59]$.

- the deviation from the principle of virtual work is written in static form at each load step $t_{i}$ using a set of virtual fields denoted $\mathbf{u}^{* j}$ and involving the measured strains and the unknown parameters $X$ through the previously computed plastic strains

$$
c_{k l}(X)=-Q_{i j} \int_{V}\left[\varepsilon_{i}\left(t_{k}\right)-\varepsilon_{i}^{\mathrm{p}}\left(X, t_{k}\right)\right] \varepsilon_{j}^{* l} \mathrm{~d} V+\int_{S_{f}} \mathbf{T}\left(t_{k}\right) \cdot \mathbf{u}^{* l} \mathrm{~d} S
$$


- $\quad$ the squares of deviations $c_{k l}(X)$ in Equation (9.2) are summed for all the virtual fields $\mathbf{u}^{* l}$ and for all the load steps $t_{k}$, yielding a cost function involving implicitly the unknown parameters

$$
\begin{aligned}
\mathcal{C}(X)= & \sum_{k=1}^{m} \sum_{l=1}^{n}\left[c_{k l}(X)\right]^{2} \\
= & \sum_{k=1}^{m} \sum_{l=1}^{n}\left[-Q_{i j} \int_{V}\left[\varepsilon_{i}\left(t_{k}\right)-\varepsilon_{i}^{\mathrm{p}}\left(X, t_{k}\right)\right]: \varepsilon_{j}^{* l} \mathrm{~d} V\right. \\
& \left.+\int_{S_{f}} \overline{\mathbf{T}}\left(t_{k}\right) \cdot \mathbf{u}^{* l} \mathrm{~d} S\right]^{2}
\end{aligned}
$$

The virtual strain fields are chosen a priori. Unknown parameters $X$ are identified by minimising $\mathcal{C}(X)$. The computation is longer than when the VFM is applied to linear elastic constitutive equations because iterative algorithms are necessary to minimise $\mathcal{C}(X)$ due to the implicit dependence on $X$.

\subsection{Experimental validation}

\subsubsection{Experimental details}

A tensile test is performed on a plane dog boneshaped specimen (Figure 10). This statically undetermined test remains quite simple because in first approximation, the longitudinal stress only varies as a function of the longitudinal axis of the specimen (non-uniform stress state) and the other stress components remain small.

The material was $99.5 \%$ pure iron. The stress-strain curves characterised with standard tensile tests were fitted by the Voce's non-linear hardening model [61]. It can be written

$\sigma^{\mathrm{VM}}=\sigma_{0}+R_{0} p+R_{\text {inf }}[1-\exp (-b p)]$

where $p$ is the cumulative plastic strain, $\sigma^{\mathrm{VM}}$ is the Von Mises stress, $\sigma_{0}$ the initial yield stress, $R_{0}$ the

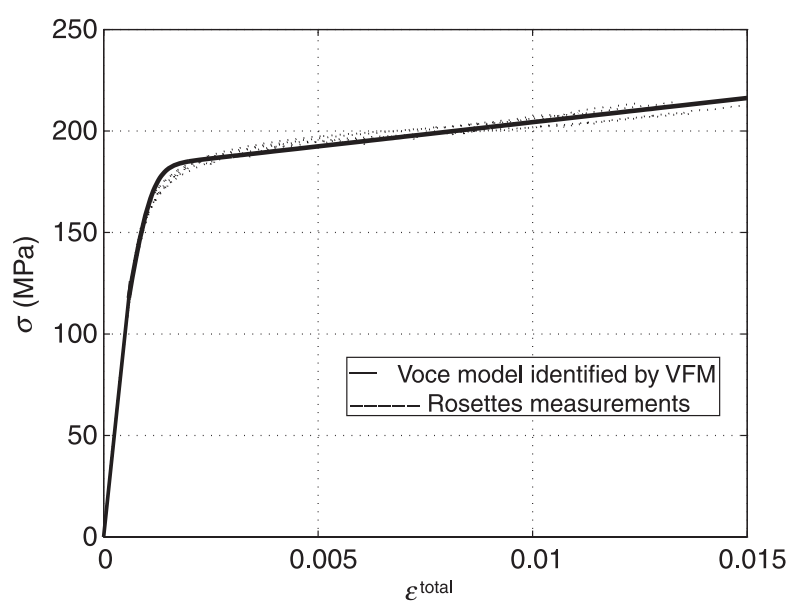

Figure I I: Comparison of rosette results with the Voce's model identified from full-field data

linear asymptotic hardening modulus, $R_{\mathrm{inf}}$ and $b$ the parameters that describe the non-linear curve part at the knee point (Figure 11). Reference values for those parameters are given in Table 5 with a confidence interval.

The full-field measurement technique used in this application is the grid method (read description in Section 6.2). However, because of the use of an imaging lens, the measurements of the in-plane displacements are sensitive to out-of-plane movements of the specimen, which are non-negligible for this test because of the testing device itself. Indeed, outof-plane movements up to $0.15 \mathrm{~mm}$ give rise to parasitic strains up to $1 \times 10^{-3}$, which is not compatible with the requirements of the experiments (strain elastic limit of about $1 \times 10^{-3}$ for this material). Therefore, a particular set-up is used to get rid of this problem. It consists in measuring the displacements on both sides of the specimen using two cameras. The cameras are positioned symmetrically with respect to the specimen plane (Figure 10). Thus, the effects of the out-of-plane movements cancel out when averaging the phase maps from both cameras.
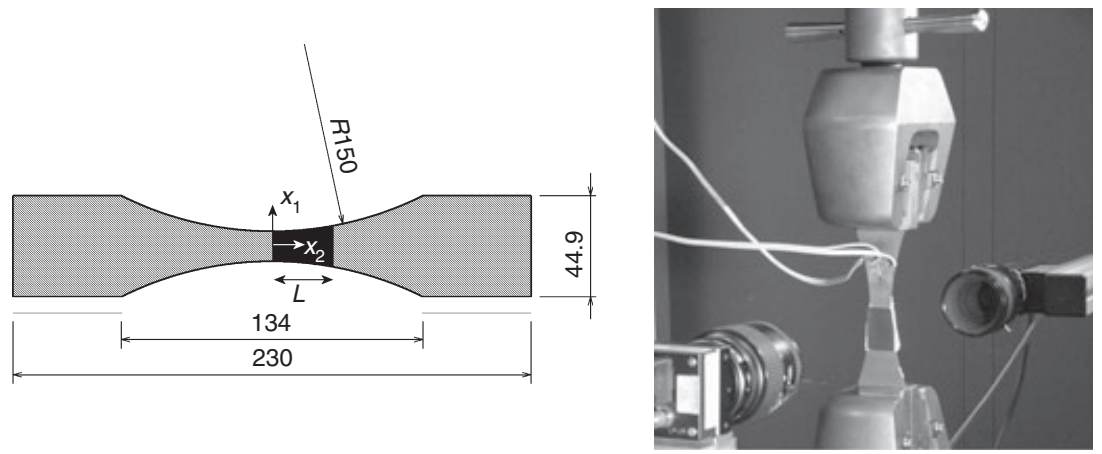

Figure 10: Specimen with mechanical and optical set-up for the application of the virtual fields method to the identification of plastic constitutive parameters 
Table 5: Comparison of the identified Voce's model parameters with reference values and cost function sensitivities

\begin{tabular}{llc}
\hline Parameter & $\begin{array}{l}\text { Reference value } \\
(\text { mean } \pm 2 \sigma)\end{array}$ & $\begin{array}{l}\text { Identified value with the } \\
\text { virtual fields method }\end{array}$ \\
\hline$\sigma_{0}$ & $127 \pm 15 \mathrm{MPa}$ & $117.5 \mathrm{MPa}$ \\
$R_{0}$ & $2.46 \pm 0.49 \mathrm{GPa}$ & $2.34 \mathrm{GPa}$ \\
$R_{\text {inf }}$ & $56.2 \pm 13.8 \mathrm{MPa}$ & $66 \mathrm{MPa}$ \\
$b$ & $3.37 \pm 0.72 \times 10^{3}$ & $5.24 \times 10^{3}$ \\
\hline
\end{tabular}

This procedure has also the advantage of accounting for possible parasitic bending strains caused by misalignment of the grips, in the same spirit as the procedure with back-to-back strain gauges used for the reference tests.

Here also, the measured displacement fields are fitted using 2D polynomials (Section 6.2). Polynomials of degree 4 are sufficient here because using further degrees do not increase the fitting accuracy. The strain field are deduced from the partial derivative of polynomials.

\subsubsection{Identification procedure}

In this application, the stress field is assumed to be plane and uniaxial. Accordingly

$$
\left\{\begin{array}{l}
\sigma_{1}=0 \\
\sigma_{2}=E\left[\varepsilon_{2}-p\left(X, t_{l}\right)\right] \\
\sigma_{6}=0
\end{array}\right.
$$

where $p\left(X, t_{l}\right)$ is the cumulative plastic strain, computed at each load step $t_{l}$ within the framework of elasto-plasticity. It involves implicitly the constitutive parameters through an iterative algorithm $[58,59]$.

The virtual fields chosen here are uniform longitudinal strain fields defined over a small length of the specimen and rigid body like over the remaining length of the area of interest (black area in Figure 10). For any of them, they can be written between abscissa $x_{2}(l)$ and $x_{2}(l+1)$

$$
\left\{\begin{array}{l}
\varepsilon_{1}^{*}=0 \\
\varepsilon_{2}^{*}=1 \\
\varepsilon_{6}^{*}=0
\end{array}\right.
$$

The area of interest of the specimen is shared over $n$ elements of equal length $L / n$ and of area $S_{l}$, yielding $n$ independent virtual fields. Finally, the cost function becomes

$\mathcal{C}\left(\sigma_{0}, R_{0}, R_{\mathrm{inf}}, b\right)=\sum_{k=1}^{m} \sum_{l=1}^{n}\left[\frac{(P L / e n)-E \int_{S_{l}}\left[\varepsilon_{2}-p\left(t_{k}, X\right)\right] \mathrm{d} S}{(P / e w) S_{l}}\right]^{2}$.
The denominator $(P / e w) S_{i}$ is introduced in order to provide dimensionless quantities; $w$ is the width of the cross-section located at the middle of the region of interest and $e$ is the thickness of the specimen.

\subsubsection{Results}

A total of 10 strain maps have been recorded (load levels: $2.6,4.3,6.5,7.8,8.2,8.7,8.9,9.2,9.5,9.7 \mathrm{kN}$ ). The first two are purely elastic. Only the eight others are processed (thus, $m=8$ in Equation 9.7).

A minimisation is performed to find the minimum of the cost function $\mathcal{C}\left(\sigma_{0}, R_{0}, R_{\mathrm{inf}}, b\right)$. [100 MPa, $1 \mathrm{GPa}, 40 \mathrm{MPa}, 1000$ ] are input as initial values in a Nelder Mead algorithm available in the Matlab software within the fminsearch command. This iterative algorithm converges after 224 iterations using the default convergence criterion of Matlab. Results are reported in Table 5 and Figure 11.

It can be noticed that the curve of the identified model plotted in Figure 11 is in good agreement with rosette data. However, this procedure obviously needs to be applied to a more complex test geometry. Experimental tests are currently on-going to validate the approach for fully multidirectional stress states.

\section{Work Underway}

The methodology presented in this paper is very general and has a great potential for a number of applications. This section aims at giving a few perspectives related to projects underway in the research groups of the authors.

\section{I0.I. Heterogeneous materials}

There are a number of situations where at the scale of the test, the material cannot be considered as homogeneous. In this case, the mechanical parameters will be dependent on the space variables and generally, the standard test methods are at a loss to address this type of problem. The variations of mechanical properties can be either the result of the manufacturing of the material (functionally graded materials, welds, etc.) or the result of some damaging phenomenon (impact, fatigue, ageing, etc.). In all cases, the assumption leading to Equation (4.3) does not hold anymore.

Two applications related to this topic are presently underway.

\subsubsection{Application to wood}

Because of the living nature of wood, the macroscopic mechanical properties vary very significantly 
from the centre to the edge and from the top to the bottom of the $\log$ [61]. As a result, small tests specimen taken at different locations in the log will give different stiffness values. If this type of procedure is used to assess this variability, as in Machado and Cruz [61], a huge number of specimens is required. Moreover, it is well known in wood mechanics that testing the properties in the plane transverse to the axis of the log (the RT plane, as it is often referred to) is extremely difficult because of the cylindrical symmetry of the log.

One possibility to address this problem would be to perform tests on large panels for which the variation of stiffness could be parameterised. For instance, let us imagine that the longitudinal modulus can be written as a quadratic function of the distance to the $\log$ central axis $r$

$Q_{l l}=Q_{l l}^{0}+\frac{\left(Q_{l l}^{R}-Q_{l l}^{0}\right)}{R^{2}} r^{2}$,

where $Q_{l l}^{0}$ is the stiffness at the centre, $Q_{l l}^{R}$ is the stiffness at the edge and $R$ is the log radius. This can now be input in Equation (4.3) and the unknown parameters $Q_{l l}^{0}$ and $Q_{l l}^{R}$ can be taken out of the integrals and identified. Obviously, many other types of parameterisation can be adopted depending on their relevance for the specific problem.

Another application concerned the validation of the procedure described in Section 6.4 to wood specimens in the LR plane. The main problem here is that at that scale, the test specimen consists of only a few growth rings and the stiffness of early and late wood is very different [62]. Therefore, the average moduli thus identified might vary depending on the constitution of the test specimen (number of growth rings, percentage of early wood). This has been explored in detail to validate the approach [63].

Finally, the last step would be to identify directly the stiffness components at the scale of the constituents, i.e. the growth rings, probably using also a continuous approach, as suggest the results in Jernkvist and Thuvander [62]. A full-field strain measurement technique using micro-grids is presently being developed to address this issue [64].

\subsubsection{Application to impacted composite plates}

Another source of heterogeneity of mechanical properties is the damage caused by some accidental loading such as impact. This problem is very important for thin composite panels used in the aerospace industry. A great number of studies have been dedicated in the past to the detection of the location of the damage using different types of techniques: infrared thermography, ultrasonic inspection, shearography, lamb waves, etc. However, these approaches can only give an indication of the location and size of damage but cannot give precise information as to the local loss of mechanical performance which is what the design office would really be interested in in order to design for damage. A recent study has been launched by the authors to try to address this issue. Thin laminated composite panels are considered here. The idea is to test the plates in bending and to measure the slope fields using a deflectometry setup such as that mentioned in Section 5 [28]. As the location of the damage is not known a priori, a discrete parameterisation of the stiffness components seems difficult as it will lead to a great number of unknowns to be identified. An alternative is to use a discrete approach by writing the bending stiffness matrix $D$ as:

$D=D^{0}\left(1+\sum_{i, j=1}^{n} a_{i j} x^{i} y^{j}\right)$,

where $D^{0}$ is the stiffness of the undamaged plate and the $a_{i j}$ are the coefficients driving the stiffness reduction. The procedure now consists in identifying these $a_{i j}$ coefficients, assuming that $D^{0}$ is known. It must be emphasised that this is a very first attempt and that a rather simple parameterisation is selected. As the project proceeds, this approach will be completed with a discrete parameterisation which is much easier to setup once the location of the damage is determined, even only approximately.

This idea has been tested with slope data obtained from finite element simulations. The test used here consists of rectangular panel supported at three corners and loaded in the middle. A carbon-epoxy crossply laminated panel has been considered and in a square area of this panel, a bending stiffness reduction $30 \%$ has been introduced by dramatically reducing the stiffness of a certain number of plies, simulating an impact damage. Then, the VFM has been applied to identify the stiffness reduction function $\sum_{i, j=1}^{n} a_{i j} x^{i} y^{j}$, with $n=5$ in this case. A map of this function is shown in Figure 12, together with the reference binary distribution that was input in the FE model (on the left). It is clear that the identification is successful both in locating the damage and in evaluating the stiffness reduction coefficient. More information can be found in Kim et al. [65].

Experimental validation of this procedure is presently underway with both artificial damage (insertion of PTFE films to simulate delamination) and real impact damage. 

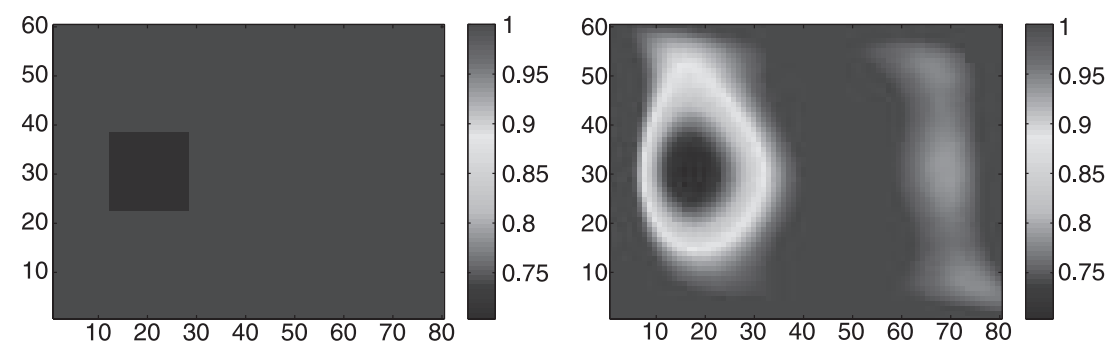

Figure 12: Reference and identified stiffness reduction coefficient on a carbon-epoxy cross-ply panel

Table 6: Identified stiffness components for both Iosipescu configurations (usual $0^{\circ}$ and optimised $-25^{\circ}$ )

\begin{tabular}{|c|c|c|c|c|c|c|c|c|}
\hline \multirow[b]{2}{*}{$\mathrm{GPa}$} & \multicolumn{4}{|c|}{$30 \mathrm{~mm}, 0^{\circ}$} & \multicolumn{4}{|c|}{$40 \mathrm{~mm},-25^{\circ}$} \\
\hline & $Q_{11}$ & $Q_{22}$ & $Q_{12}$ & $Q_{66}$ & $Q_{11}$ & $Q_{22}$ & $Q_{12}$ & $Q_{66}$ \\
\hline Test I & 43.8 & 4.00 & 2.72 & 3.47 & 48.3 & 9.41 & 2.98 & 3.94 \\
\hline Test 2 & 45.7 & 5.88 & 3.36 & 3.57 & 46.7 & 9.26 & 2.35 & 4.04 \\
\hline Test 3 & 48.1 & 6.73 & 3.33 & 3.40 & 44.2 & 9.15 & 1.93 & 4.38 \\
\hline Test 4 & 47.4 & 6.60 & 3.26 & 3.53 & 45.9 & 8.92 & 2.54 & 3.82 \\
\hline Test 5 & 48.8 & 6.89 & 3.69 & 3.48 & 48.4 & 8.26 & 2.27 & 3.61 \\
\hline Test 6 & & & & & 47.4 & 9.25 & 2.85 & 3.84 \\
\hline Reference & 44.9 & 12.2 & 3.86 & 3.68 & 44.9 & 12.2 & 3.86 & 3.68 \\
\hline Mean & 46.8 & 6.02 & 3.27 & 3.49 & 46.8 & 9.04 & 2.49 & 3.94 \\
\hline $\begin{array}{l}\text { Coefficient of } \\
\text { variation (\%) }\end{array}$ & 4.3 & 19.9 & 10.7 & 1.9 & 3.3 & 4.6 & 15.5 & 6.6 \\
\hline
\end{tabular}

\subsection{Optimisation of test configurations}

Another very important issue in this field is the design of appropriate test configurations. Indeed, most of the existing tests have been designed with a view to obtain uniform and simple stress and strain fields. When the VFM is used, the tests must on the contrary exhibit non-uniform distributions and, if possible, balanced stress contributions. The restrictions on shape and loading arrangements are greatly reduced and a very large design space can be explored. Only very few attempts have be made in the literature to optimise the design configuration of tests based on full-field interpretation. For instance, Le Magorou et al. [8] optimised the locations of support points and loading points to balance the contribution of the different stiffness components on the response of their panel tested in bending. As the inverse identification method they use is finite element model updating, the cost function used for the optimisation involves finite element calculated sensitivities. Recent work by some of the authors shows that it is possible to perform a similar procedure but adapted to the VFM [66].

Another attempt has also been made at optimising the unnotched Iosipescu specimen presented in Section 6.4. The idea here was to use the active gauge length $L$ and the fibre orientation $\theta$ as design variables in order to minimise the following cost function

$\phi(L, \theta)=\frac{\left(\xi_{x}-\xi_{y}\right)^{2}+\left(\xi_{x}-\xi_{s}\right)^{2}+\left(\xi_{y}-\xi_{s}\right)^{2}}{3\left(\xi_{x}^{2}+\xi_{y}^{2}+\xi_{s}^{2}\right)}$

with

$\xi_{\alpha}=\frac{\eta_{\alpha}}{Q_{\alpha \alpha}}, \quad \alpha=x, y, s$,

where $\eta_{x}, \eta_{y}$ and $\eta_{s}$ are the coefficients of sensitivity to noise related to respectively $Q_{11}, Q_{22}$ and $Q_{66}$ [68]. Therefore, the cost function will be minimal when these three stiffness components will be identified with the same confidence. Dividing by the sum of the coefficients is a normalisation procedure to keep the cost function independent of the actual strain values (i.e. independent of the force applied). This procedure has been implemented by connecting a finite element package (here, Ansys) to the virtual fields routine written in Matlab. A parametric finite element model is written in the form of an input ASCII file, fed into the FE package and the strain results are then processed by the VFM routine. The $\eta$ parameters are then calculated and the cost function evaluated. The FE input ASCII file is then updated with new values of the design variables (length and angle) and the routine run again. The optimisation routine has been run automatically from Matlab using a Nelder Mead minimisation algorithm implemented in Matlab (fminsearch function). The procedure has been launched with different initial values of the design variables and several solutions have been found. One of these solutions is $L=40 \mathrm{~mm}$ and $\theta=-25^{\circ}$. This configuration has been tested and compared with the usual $L=30 \mathrm{~mm}$ and $\theta=0^{\circ}$ on the same glass-epoxy material as that of Section 6.4. The measurements have been performed at Airbus UK in Filton using speckle interferometry and the same Iosipescu configuration as that of Section 6.4. The data processing is the same as that presented in Chalal et al. [43], except that the displacement fields are fitted by 7th order polynomials in order to derive the strains [48]. 
One specimen of each configuration has been tested several times. The results have been compared in terms of average and standard deviation. The results are reported in Table 6 [67].

It is very interesting to note that for the optimised configuration, the coefficients of variation of the target stiffness components of the cost function (all except $Q_{12}$ ) are now of the same order (a few percents), which was not the case for the $0^{\circ}$ configuration. Also, as a result, the transverse stiffness $Q_{22}$ is much better identified with the $-25^{\circ}$ than with the $0^{\circ}$ configuration. $Q_{12}$ remains to be improved but this parameter is always more difficult to identify as its influence on the actual strain field is smaller than for the other components (which was the reason why is was left out in the cost function). This very first result is very promising and more work along this line is presently underway.

\section{Conclusion}

The main aspects of the VFM are presented in this paper. The ability to extract constitutive parameters from full-field measurements is illustrated through various examples. One of the main features of the method is to mix in the weak form of the equilibrium condition some virtual fields which have to be chosen by the user and actual measured fields provided by a full-field measurement technique. These field data are in fact the input of the method. It is shown that this leads to the direct extraction of the parameters in the case of constitutive equations depending linearly on the constitutive parameters whereas some iterative calculations are required in the case of non-linear equations. The choice of the virtual fields is a key issue when using this approach. It has been thoroughly addressed in the recent past in the case of linear constitutive equations. This has first led to the definition of so-called special virtual fields and then to a choice of these special virtual fields which reduces the influence of noisy data on identified parameters. The use of the method to non-linear equations is more recent and the question of the optimal choice of the virtual fields in this case remains clearly open at this stage. More complicated non-linear equations should also be examined to assess the applicability of the VFM in these cases. It must also be underlined that processing heterogeneous strain fields gives an extra freedom in the design of the mechanical tests in terms of specimen shape and definition of the loading as the requirement of homogeneous tests is avoided. This feature is somewhat troublesome as heterogeneity is no longer a drawback but becomes here an advantage. Taking full advantage of this extra freedom is another important issue which has not been investigated in details till now. Lateral thinking will be required for the development of more complex mechanical test fully optimised to the VFM. This is probably one of the main challenges which has to be faced in the near future to go further with this method.

\section{ACKNOWLEDGEMENTS}

The authors would like to thank all their colleagues and students who have been involved in the development of the VFM over the years. Their names appear as co-authors of the many papers cited in reference. A number of institutional funding bodies have also greatly contributed to this task and the present authors would like to thank them: Champagne Ardenne Regional Council, French Ministry for Research (PhD thesis grants, ACI research grant) and the French National Research Council (CNRS) through the GDR 2519 collaborative research group, to cite only the most important ones. The authors would also like to thank Airbus UK, Filton, and particularly Dr Richard Burguete, for supporting the study on optimisation of the Iosipescu configuration.

\section{REFERENCES}

1. Pindera, M.-J. and Herakovich, C. T. (1986) Shear characterization of unidirectional composites with the off-axis tensile test. Exp. Mech. 26, 103-112.

2. Pierron, F., Alloba, E., Surrel, Y. and Vautrin, A. (1998) Whole-field assessment of the effects of boundary conditions on the strain field in off-axis tensile testing of unidirectional composites. Compos. Sci. Technol. 58, 19391947.

3. Hendricks, M. A. N. (1991) Identification of the mechanical properties of solid materials. Doctoral Dissertation, Eindhoven University of Technology, Eindhoven, The Netherlands.

4. Geymonat, G., Hild, F. and Pagano, S. (2002) Identification of elastic parameters by displacement field measurement. C. R. Acad. Sci. 330, 403-408.

5. Claire, D., Hild, F. and Roux, S. (2002) Identification of damage fields using kinematic measurements. C. R. Acad. Sci. 330, 729-734.

6. Ikehata, M. (1990) Inversion formulas for the linearized problem for an inverse boundary value problem in elastic prospection. SIAM J. Appl. Math. 50, 1635-1644.

7. Grédiac, M. (1989) Principe des travaux virtuels et identification. C. R. Acad. Sci. 309, 1-5 (In French with abridged English version).

8. LeMagorou, L., Bos, F. and Rouger, F. (2002) Identification of constitutive laws for wood-based panels by means of an inverse method. Compos. Sci. Technol. 62 , 591-596. 
9. Mahnken, R. and Stein, E. (1994) The identification of parameters for visco-plastic models via finite elements methods and gradient methods. Model. Simulat. Mater. Sci. Eng. 2, 597-616.

10. Mahnken, R. and Stein, E. (1996) A unified approach for parameter identification of inelastic material models in the frame of the finite element method. Comput. Methods Appl. Mech. Eng. 136, 225-258.

11. Meuwissen, M. H. H. (1998) An inverse method for the mechanical characterisation of metals. PhD Thesis, Eindhoven Technical University, Eindhoven, The Netherlands.

12. Meuwissen, M. H. H., Oomens, C. W. J., Baaijens, F. P. T., Petterson, R. and Janssen, J. D. (1998) Determination of the elasto-plastic properties of aluminium using a mixed numerical-experimental method. J. Mater. Process. Technol. $75,204-211$.

13. Okada, H., Fukui, Y. and Kumazawa, N. (1999) An inverse analysis determining the elastic-plastic stress-strain relationship using nonlinear sensitivities. Comput. Model. Simulat. Eng. 4, 176-185.

14. Hoc, T., Crépin, J., Gélébart, L. and Zaoui, A. (2003) A procedure for identifying the plastic behaviour of single crystals from the local response of polycrystals. Acta Mater. 51, 5477-5488.

15. Kajberg, J. and Lindkvist, G. (2004) Characterization of materials subjected to large strains by inverse modelling based on in-plane displacement fields. Int. J. Solids Struct. 41, 3439-3459.

16. Geng, L., Shen, Y. and Wagoner, R. H. (2002) Anisotropic hardening equations derived from reversebend testing. Int. J. Plasticity 18, 743-767.

17. Dym, C. L. and Shames, I. H. (1973) Solid Mechanics. A Variational Approach. McGraw-Hill Book Co., Tokyo.

18. Grédiac, M., Toussaint, E. and Pierron, F. (2002) Special virtual fields for the direct determination of material parameters with the virtual fields method. 1 - Principle and definition. Int. J. Solids Struct. 39, 2691-2705.

19. Golub, G. H. and Van Loan, C. F. (1993) Matrix Computations, 3rd edn. The Johns Hopkins University Press, Baltimore, MD.

20. Grédiac, M. and Vautrin, A. (1990) A new method for determination of bending rigidities of thin anisotropic plates. J. Appl. Mech. 57, 964-968 (Transactions of the American Society of Mechanical Engineers).

21. Grédiac, M., Toussaint, E. and Pierron, F. (2002) Special virtual fields for the direct determination of material parameters with the virtual fields method. 2 - Application to in-plane properties. Int. J. Solids Struct. 39, 2707-2730.

22. Soong, T. T. and Grigoriu, M. (1993) Random Vibration of Mechanical and Structural Systems. PTR Prentice Hall, Inc., Upper Saddle River, NJ.

23. Avril, S., Grédiac, M. and Pierron, F. (2004) Sensitivity of the virtual fields method to noisy data. Comput. Mech. 34, 439-452.

24. Timoshenko, S. and Woinowsky-Krieger, S. (1959) Theory of Plates and Shells. McGraw-Hill Book Co., Singapore.

25. Grédiac, M. (1996) On the direct determination of invariant parameters governing the bending of anisotropic plates. Int. J. Solids Struct. 33, 3969-3982.
26. Grédiac, M. and Vautrin, A. (1993) Mechanical characterization of anisotropic plates: experiments and results. Eur. J. Mech. A Solids 12, 819-838.

27. Grédiac, M., Toussaint, E. and Pierron, F. (2003) Special virtual fields for the direct determination of material parameters with the virtual fields method. 3 - Application to the bending rigidities of anisotropic plates. Int. J. Solids Struct. 40, 2401-2419.

28. Surrel, Y., Fournier, N., Grédiac, M. and Paris, P.-A. (1999) Phase-stepped deflectometry applied to shape measurement of bent plates. Exp. Mech. 39, 66-70.

29. Bruno, L. and Poggialini, A. (2005) Elastic characterization of anisotropic materials by speckle interferometry. J. Appl. Mech. 45, 205-212.

30. Grédiac, M., Fournier, N., Paris, P.-A. and Surrel, Y. (1999) Direct measurement of invariant parameters of composite plates. J. Compos. Mater. 33, 1939-1965.

31. Grédiac, M. and Paris, P.-A. (1996) Direct identification of elastic constants of anisotropic plates by modal analysis: theoretical and numerical aspects. J. Sound Vibrat. 195, 401-415.

32. Grédiac, M., Fournier, N., Paris, P.-A. and Surrel, Y. (1998) Direct identification of elastic constants of anisotropic plates by modal analysis: experiments and results. J. Sound Vibrat. 210, 645-659.

33. ASTM D5379-93 (1993) Standard Method for Shear Properties of Composite Materials by the V-notched Beam Method. American Society for the Testing of Materials, West Conshohocken, PA.

34. Grédiac, M., Pierron, F. and Vautrin, A. (1994) The Iosipescu in-plane shear test applied to composites: a new approach based on displacement field processing. Compos. Sci. Technol. 51, 409-417.

35. Grédiac, M. and Pierron, F. (1998) A T-shaped specimen for the direct characterization of orthotropic materials. Int. J. Numer. Methods Eng. 41, 293-309.

36. Grédiac, M., Pierron, F. and Surrel, Y. (1999) Novel procedure for complete in-plane composite characterization using a single T-shaped specimen. Exp. Mech. 39, 142-149.

37. Grédiac, M. and Pierron, F. (2004) Numerical issues in the virtual fields method. Int. J. Numer. Methods Eng. 59, 12871312.

38. Surrel, Y. (1994) Moiré and grid methods: a signal processing approach. In: Interferometry '94: Photomechanics, Vol. 2342 (J. Pryputniewicz and R. J. et Stupnicki, Eds). The International Society for Optical Engineering, SPIE, Bellingham, WA: 213-220.

39. Surrel, Y. (1996) Design of algorithms for phase measurement by the use of phase stepping. Appl. Optics 35, 5160.

40. Avril, S., Vautrin, A. and Surrel, Y. (2004) Grid method: application to the characterization of cracks. Exp. Mech. 44, 37-43.

41. Piro, J.-L. and Grédiac, M. (2004) Producing and transferring low-spatial-frequency grids for measuring displacement fields with moiré and grid methods. Exp. Tech. 28, 23-26.

42. Surrel, Y. (1999) Fringe analysis. In: Photomechanics (P. K. Rastogi, Ed.). Springer-Verlag, Berlin: 57-104. 
43. Chalal, H., Avril, S., Pierron, F. and Meraghni, F. (2006) Experimental identification of a damage model for composites using the grid technique coupled to the virtual fields method. Compos. A Appl. Sci. Manufact. 37, 315-325.

44. Lira, I., Cordero, R., François, M. and Vial-Edwards, C. (2004) The uncertainty of experimental derivatives: application to strain measurement. Measure. Sci. Technol. 15, 2381-2388.

45. Pierron, F., Zhavoronok, S. and Grédiac, M. (2000) Identification of the through-thickness properties of thick laminates using the virtual fields method. Int. J. Solids Struct. 37, 4437-4453.

46. Moulart, R., Avril, S. and Pierron, F. (2006) Identification of the through-thickness rigidities of a thick laminated composite tube. Compos. A Appl. Sci. Manufact. 37, 326336.

47. Pierron, F. and Grédiac, M. (2000) Identification of the through-thickness moduli of thick composites from whole-field measurements using the Iosipescu fixture: theory and simulations. Compos. A Appl. Sci. Manufact. 31, 309-318.

48. Chalal, H., Avril, S. and Pierron, F. (2005) Characterization of the nonlinear shear behaviour of UD composite materials using the Virtual Fields Method. Fourth International Conference on Advances in Experimental Mechanics, British Society for Strain Measurement, Southampton, UK. CDRom of the Proceedings.

49. Grédiac, M., Auslender, F. and Pierron, F. (2001) Using the virtual fields method to identify the through-thickness moduli of thick composites with a nonlinear shear response. Compos. A Appl. Sci. Manufact. 32, 1713-1725.

50. Chalal, H., Meraghni, F., Pierron, F. and Grédiac, M. (2004) Direct identification of the damage behaviour of composite materials using the virtual fields method. Compos. A Appl. Sci. Manufact. 35, 841-848.

51. Ladevèze, P. and Le Dantec, E. (1992) Damage modeling of the elementary ply for laminated composites. Compos. Sci. Technol. 43, 257-267.

52. Giraudeau, A., Pierron, F. and Chambard, J.-P. (2002) Experimental study of air effect on vibrating lightweight structures. SEM Annual Congress on Experimental Mechanics. Society for Experimental Mechanics, Milwaukee, WI: CDRom of the Proceedings.

53. Giraudeau, A. and Pierron, F. (2003) Simultaneous identification of stiffness and damping properties of isotropic materials from forced vibrating plates. C. R. Méc. 331, 259-264.

54. Giraudeau, A. and Pierron, F. (2005) Identification of stiffness and damping properties of thin isotropic vibrating plates using the virtual fields method. Theory and simulations. J. Sound Vibrat. 284, 757-781.

55. Surrel, Y. (2004) Deflectometry: a simple and efficient noninterferometric method for slope measurement. Xth
SEM International Congress on Experimental Mechanics. Society for Experimental Mechanics, Costa Mesa, CA: CDRom of the Proceedings.

56. Giraudeau, A., Guo, B. and Pierron, F. (2005) Experimental application of the virtual fields method to the identification of stiffness and damping material properties using vibrating plates. Fourth International Conference on Advances in Experimental Mechanics. British Society for Strain Measurement, Southampton, UK: CDRom of the Proceedings.

57. Grediac, M. and Pierron, F. (2005) Applying the virtual field method to the identification of elastoplastic constitutive parameters. Int. J. Plasticity, 26, 602-627.

58. Pannier, Y., Rotinat, R., Avril, S. and Pierron, F. (2005) Heterogeneous test for elastoplastic identification by the virtual fields method. SEM Conference and Exhibition. Society for Experimental Mechanics, Portland, OR: CDRom of the Proceedings.

59. Sutton, M. A., Deng, X., Liu, J. and Yang, L. (1996) Determination of elastic plastic stresses and strains from measured surface strain data. Exp. Mech. 36, 99-112.

60. Voce, E. (1955) A practical strain hardening function. Metallurgia 51, 219.

61. Machado, J. S. and Cruz, H. P. (2005) Within stem variations of Maritime Pine timber mechanical properties. Holz als Roh- und Werkstoff 63, 154-159.

62. Jernkvist, L. O. and Thuvander, F. (2001) Experimental determination of stiffness variation across growth rings in Picea abies. Holzforschung 55, 309-317.

63. Xavier, J., Avril, S., Pierron, F. and Morais, J. (2005) Identification of clear wood anisotropic stiffness using full-field measurements. 8th European Mechanics of Materials Conference (EMMC8). Cachan, France: 45-52.

64. Moulart, R., Rotinat, R., Pierron, F. and Lerondel, G. (2006) Characterization of the mechanical behaviour of materials at a microscopic scale: development of an optical full-field measurement method. Photomechanics 2006. Clermont-Ferrand, France: 117-118.

65. Kim, J., Pierron, F., Syed-Muhammad, K., Wisnom, M., Grédiac, M. and Toussaint, E. (2006) Assessment of the local stiffness reduction on a composite impacted plate with the virtual fields method. Photomechanics 2006. Clermont-Ferrand, France: 58-59.

66. Syed-Muhammad, K., Toussaint, E., Grédiac, M. and Avril, S. (2005) Characterization of composite plates using the virtual fields method: piecewise virtual fields, minimization of noise effect and optimization of testing configuration. 8th European Mechanics of Materials Conference (EMMC8). Cachan, France: 177-184.

67. Pierron, F., Vert, G., Burguete, R., Avril, S., Rotinat, R. and Wisnom, M. (2006) Identification of orthotropic stiffnesses of composites with the virtual fields method: optimization and experimental validation. Photomechanics 2006. Clermont-Ferrand, France: 137-138. 